\title{
Critical Points of the Distance to an epsilon-Sampling of a Surface and Flow-Complex-Based Surface Reconstruction
}

\author{
Tamal K. Dey* \\ Department of CSE \\ The Ohio State University \\ Columbus, OH 43210 \\ tamaldey@cse.ohio-state.edu
}

\author{
Joachim Giesen $^{\dagger}$ \\ Theoretische Informatik \\ ETH Zürich \\ CH-8092 Zürich \\ giesen@inf.ethz.ch
}

\author{
Edgar A. Ramos \\ Dept. of Computer Science \\ University of Illinois \\ Urbana, IL 61801 \\ eramosn@cs.uiuc.edu
}

\author{
Bardia Sadri \\ Dept. of Computer Science \\ University of Illinois \\ Urbana, IL 61801 \\ sadri@cs.uiuc.edu
}

\begin{abstract}
The distance function to surfaces in three dimensions plays a key role in many geometric modeling applications such as medial axis approximations, surface reconstructions, offset computations, feature extractions and others. In most cases, the distance function induced by the surface is approximated by a discrete distance function induced by a discrete sample of the surface. The critical points of the distance function determine the topology of the set inducing the function. However, no earlier theoretical result has linked the critical points of the distance to a sampling of geometric structures to their topological properties. We provide this link by showing that the critical points of the distance function induced by a discrete sample of a surface either lie very close to the surface or near its medial axis and this closeness is quantified with the sampling density. Based on this result, we provide a new flow-complex-based surface reconstruction algorithm that, given a tight $\varepsilon$-sampling of a surface, approximates the surface geometrically, both in Hausdorff distance and normals, and captures its topology.
\end{abstract}

\section{INTRODUCTION}

Given a compact surface $\Sigma$ smoothly embedded in three dimensional Euclidean space $\mathbb{R}^{3}$, a distance function

$$
h_{\Sigma}: \mathbb{R}^{3} \rightarrow \mathbb{R}, x \mapsto \inf _{p \in \Sigma}\|x-p\| .
$$

can be defined over $\mathbb{R}^{3}$ that assigns to each point its distance to $\Sigma$. This distance function carries a lot of information about $\Sigma$ and its embedding. The surface itself can be

\footnotetext{
*Research partly supported by NSF grants DMS-0138456, DMS0310642 and ARO grant DAAD19-02-1-0347.

${ }^{\dagger}$ Research partially supported by the Swiss National Science Foundation under the project "Non-linear manifold learning".
}

Permission to make digital or hard copies of all or part of this work for personal or classroom use is granted without fee provided that copies are not made or distributed for profit or commercial advantage and that copies bear this notice and the full citation on the first page. To copy otherwise, to republish, to post on servers or to redistribute to lists, requires prior specific permission and/or a fee.

Copyright 200X ACM X-XXXXX-XX-X/XX/XX $\ldots \$ 5.00$. determined as $h_{\Sigma}^{-1}(0)$ and the embedding of $\Sigma$ into $\mathbb{R}^{3}$ is essentially encoded in the medial axis $M$ of $\Sigma$ which consists of all points in $\mathbb{R}^{3}$ at which $h_{\Sigma}$ is not differentiable. For example, the homotopy type of the medial axis of an embedded torus is useful to decide if the embedding of the torus is knotted or not.

In applications $\Sigma$ is often only known via a finite sample $P$ of $\Sigma$ from which one desires to learn about $\Sigma$ and its embedding. The famous $\varepsilon$-sampling theory of Amenta and Bern [1] provides a framework to analyze algorithms that either reconstruct $\Sigma$ from $P$ or approximate the medial axis of $\Sigma$. Given an $\varepsilon$-sample of $\Sigma$ for a certain value of $\varepsilon$, the algorithms of Amenta and Bern [1], Amenta, Choi, Dey and Leekha [3] and Boissonnat and Cazals [5] allow to reconstruct the correct topology of $\Sigma$ and to approximate its geometry in terms of $\varepsilon$. On the other hand the algorithms of Amenta, Choi and Kolluri [4] allows to reconstruct the homotopy type of the medial axis $M$ correctly whereas the algorithm of Dey and Zhao [10] approximates the geometry of $M$ in terms of $\varepsilon$.

It is quite natural to try to approximate the distance function $h_{\Sigma}$ by the function

$$
h_{P}: \mathbb{R}^{3} \rightarrow \mathbb{R}, x \mapsto \min _{p \in P}\|x-p\| .
$$

over $\mathbb{R}^{3}$ that assigns to each point its distance to the sample $P$ of $\Sigma$. This approximation has been used by Edelsbrunner [11], Chaine [6], and Giesen and John [12] to reconstruct $\Sigma$ from $P$. Though all three of these algorithms work well in practice no guarantees for the geometric quality of their output in the $\varepsilon$-sampling framework existed until now. The critical points of $h_{P}$ were also used by Dey, Giesen and Goswami [7] to segment the solid bounded by $\Sigma$ into its features.

All surface reconstruction algorithms based on $h_{P}$ make use of its critical points, i.e., its local extrema and saddle points. These points are easily computable from the Delaunay triangulation of $P$. A first contribution of our paper is to relate these critical points for an $\varepsilon$-sampling of $\Sigma$ to both $\Sigma$ itself and its medial axis $M$. The distance functions $h_{\Sigma}$ and $h_{P}$ are not smooth everywhere. Nevertheless, there is a well developed theory of critical points of such functions [13]. The critical points of $h_{\Sigma}$ are all points in $\Sigma$ and a subset of the medial axis $M$. For example, all local maxima and all saddle points of $h_{\Sigma}$ are on $M$. We can show that for an $\varepsilon$-sampling 
for a certain value of $\varepsilon$ all critical points of $h_{P}$ either reside very close to $\Sigma$ or close to $M$. That is, we can label the critical points of $h_{P}$ as either surface critical points if they are close to $\Sigma$ or medial axis critical points if they are close to $M$. Interestingly, all types of critical points, including local maxima, can be close to $\Sigma$. The separation of the critical points is also remarkable in the following sense: The medial axis of $P$ is the 2 -skeleton of the Voronoi diagram of $P$. But not every facet in the 2 -skeleton can be assigned unambiguously to either $\Sigma$ or $M$ even if $\varepsilon$ becomes arbitrarily small. It is well know that Voronoi vertices can reside almost anywhere in $\mathbb{R}^{3} \backslash \Sigma$.

The separation of the critical points of $h_{P}$ can be turned into an algorithm to reconstruct $\Sigma$ from $P$. This is the second main contribution of our paper. We can show that the reconstructed surface is homeomorphic to $\Sigma$ and geometrically close to it both in Hausdorff distance and deviations of normals provided the input is a tight $\varepsilon$-sample of $\Sigma$. Similar results hold for curves and curve reconstruction.

The structure of the paper is as follows. Section 2 introduces the basic concepts including flow complex and critical points of the distance function of a point set. In section 3, we show that the critical points of the distance function of an $\varepsilon$-sampling of a surface are either close to the surface or close to its medial axis. Section 4 describes how it can be algorithmically determined for a critical point whether it is close to the surface or to the medial axis and uses this to build a surface reconstruction algorithm. Section 5 analyzes the quality of the produced reconstruction and establishes its geometric closeness and topological correctness. Finally, Section 6 studies the critical points of a smooth curve in $\mathbb{R}^{3}$ and gives algorithms for classification of critical points and reconstruction of the curve analogous to those of the surface. Concluding remarks are given in Section 7.

\section{BASIC CONCEPTS}

Throughout the paper, by $\Sigma$, we refer to a smooth 2-manifold without boundary embedded in $\mathbb{R}^{3}$. Since it does not have a boundary, $\Sigma$ separates $\mathbb{R}^{3}$ into a bounded region and an unbounded region. With a slight abuse of terminology we refer to the bounded region as the interior of $\Sigma$ and to the unbounded region as its exterior. Since $\Sigma$ is smooth, the normal to $\Sigma$ at any point $x \in \Sigma$ is well defined. For $x \in \Sigma$, we denote by $n_{x}^{+}$and $n_{x}^{-}$, the normal vectors at $x$ pointing to the exterior and interior of $\Sigma$ respectively. By $n_{x}$ (with no + or - superscript) we denote either of $n_{x}^{+}$or $n_{x}^{-}$, i.e. the unoriented direction of the line normal to $\Sigma$ at $x$. We also denote throughout by $P \subset \Sigma$ a discrete sample satisfying certain conditions to be specified shortly. To simplify our exposition we assume that $P$ is in general position.

Any point set $S \subset \mathbb{R}^{3}$ induces a distance function

$$
h_{S}: \mathbb{R}^{3} \rightarrow \mathbb{R}, x \mapsto \inf _{p \in S}\|x-p\|,
$$

where $\|\cdot\|$ denotes the Euclidean norm. It is easy to check that every distance function in the above sense is Lipschitz, i.e. for all $x, y \in \mathbb{R}^{3},\left|h_{S}(x)-h_{S}(y)\right| \leq\|x-y\|$. In this paper, we work with two major distance functions, one induced by $\Sigma$ and the other by $P$. To simplify our notation, in the sequel, we use $s(\cdot)$ instead of $h_{\Sigma}(\cdot)$ and $h(\cdot)$ instead $h_{P}(\cdot)$.

\subsection{Surface samples}

The medial axis $M=M(\Sigma)$ of $\Sigma$ is the set of all points in $\mathbb{R}^{3}$ that have at least 2 distinct closest points in $\Sigma$, i.e.

$$
M=\left\{x \in \mathbb{R}^{3}:\left|\left\{y \in \Sigma:\|x-y\|=h_{\Sigma}(x)\right\}\right| \geq 2\right\} .
$$

For a point $c \in \mathbb{R}^{3}$ and real number $r$, the ball with center $c$ and radius $r$, denoted $B_{c, r}$, is the set of all points $x \in \mathbb{R}^{3}$ at distance no more than $r$ from $c$. We call a ball empty, if its interior does not contain any point from $\Sigma$. A medial ball is a maximal empty ball, i.e. an empty ball that is not contained in any other empty ball.

Medial feature size. For any point $x \in \mathbb{R}^{3} \backslash(\Sigma \cup M)$ we denote by $\hat{x}$ the unique closest surface point to $x$, i.e.,

$$
\hat{x}=\operatorname{argmin}_{y \in \Sigma}\|x-y\|,
$$

and by $\check{x} \in M$ we denote the center of the medial ball tangent to $\Sigma$ at $\hat{x}$ and at the same side of $\Sigma$ as $x$. The medial feature size is the function

$$
\mu: \mathbb{R}^{3} \backslash(\Sigma \cup M) \rightarrow \mathbb{R} \cup\{\infty\}, x \mapsto\|\hat{x}-\check{x}\| .
$$

Besides the medial feature size we will also use the function

$$
m: \mathbb{R}^{3} \backslash(\Sigma \cup M) \rightarrow \mathbb{R} \cup\{\infty\}, x \mapsto\|x-\check{x}\|,
$$

which we refer to as the medial projection length. Notice that for every $x \in \mathbb{R}^{3} \backslash(\Sigma \cup M)$ we have the identity $\mu(x)=$ $m(x)+s(x)$.

Feature size. The function

$$
f: \Sigma \rightarrow \mathbb{R}^{3}, x \mapsto \inf _{y \in M}\|x-y\|
$$

which assigns to each point in $\Sigma$ its distance to the medial axis $M$, is called the local feature size. Notice that for $x \in$ $\mathbb{R}^{3} \backslash(\Sigma \cup M)$ it always holds that $f(\hat{x}) \leq \mu(x)$. Notice also that $f(\cdot)$ is also a distance function and therefore Lipschitz.

Sampling conditions. For a constant $\varepsilon>0$, a finite sample $P \subset \Sigma$ is called an $\varepsilon$-sample if

$$
\forall x \in \Sigma \exists p \in P \text { such that }\|x-p\| \leq \varepsilon f(x) .
$$

An $\varepsilon$-sample $P$ is called an $(\varepsilon, \delta)$-sample or a tight $\varepsilon$-sample if it satisfies the additional condition

$$
\forall p, q \in P \text { it holds that }\|p-q\| \geq \delta f(p)
$$

for some $\delta$, with $0<\delta<\varepsilon$.

Poles. For a sample point $p \in P$ we denote by $V_{p}$ the closed Voronoi cell of $p$. If $V_{p}$ is bounded, the positive pole of $p$, denoted $p^{+}$, is the Voronoi vertex of $V_{p}$ farthest away from $p$. The positive pole vector $\nu_{p}^{+}$is the vector $p^{+}-p$ if $V_{p}$ is bounded or is taken as the unit vector in the direction which is the average of all unbounded Voronoi edges in $V_{p}$. In the latter case we informally refer to a point at infinity in the direction $\nu_{p}^{+}$as the positive pole. The negative pole $p^{-}$of $p$ is the farthest Voronoi vertex of $V_{p}$ from $p$ for which the acute angle between the vectors $\nu_{p}^{+}$and $\nu_{p}^{-}=p^{-}-p$ is greater than than $\pi / 2$. We call $\nu_{p}^{-}$the negative pole vector of $p$.

Notation. The angle between two vectors $u$ and $v$, denoted $\angle(u, v)$ is always smaller than $\pi$. For three points $x, y$, and $z$, we denote by $\angle x y z$ the angle between vectors $x-y$ and $z-y$, i.e., $\angle(x-y, z-y)$. The acute angle between vectors 
$u$ and $v$ is the smaller of the two angles made by the lines through $u$ and $v$. The latter angle is at most $\pi / 2$.

We use the following two lemmas due to Amenta and Bern [1] and the corollary below them several times in this paper.

LEMmA 1. Let $x$ and $y$ be points on $\Sigma$ with $\|x-y\| \leq$ $\xi f(x)$ for $\xi \leq 1 / 3$. Then $\angle\left(n_{x}^{+}, n_{y}^{+}\right)=\angle\left(n_{x}^{-}, n_{y}^{-}\right) \leq \frac{\xi}{1-3 \xi}$.

Lemma 2. Let $p$ be a sample point in an $\varepsilon$-sample P. Let $x$ be any point in $V_{p}$ with $\|x-p\| \geq \xi f(p)$ for $\xi>0$. Then $\angle\left(x-p, n_{p}\right) \leq \arcsin \frac{\varepsilon}{1-\varepsilon}+\arcsin \frac{\varepsilon}{\xi(1-\varepsilon)}$.

COROllary 1. For any point $p$ of an $\varepsilon$-sampling $P$ of a surface $\Sigma$, the acute angle between $n_{p}$, normal to surface at $p$, and either of $\nu_{p}^{+}$and $\nu_{p}^{-}$is at most $2 \arcsin (\varepsilon /(1-\varepsilon))$.

\subsection{Induced flows}

Critical points. We are interested in the critical points of $h$, i.e., its local extrema and saddle points. In general, a point $c \in \mathbb{R}^{3}$ is a critical point of a distance function $h_{S}$ induced by a set $S$, if $c$ is contained in the convex hull of its closest points in $S$. Thus if we let for every $x \in \mathbb{R}^{3}$

$$
A(x)=\{p \in P:\|x-p\|=h(x)\}
$$

be the set of closest sample points to $x$, a critical point of $h$ is any point $c$ satisfying $c \in \operatorname{conv} A(c)$. It turns out that these points are exactly the intersection points of Voronoi faces and their dual Delaunay simplices. The local maxima are Voronoi vertices contained in their dual Delaunay tetrahedron. All sample points are minima. The remaining critical points are saddle points. In the case that $P$ is finite one can even assign a meaningful index to a critical point, namely, the dimension of the Delaunay simplex it is contained in.

Flow. As in the case of smooth functions there is a unique direction of steepest ascent of $h$ at every non-critical point of $h$. The direction of steepest ascent at $x \in \mathbb{R}^{3}$ is given by the vector from $x-d(x)$ where

$$
d(x)=\operatorname{argmin}_{y \in \operatorname{conv} A(x)}\|x-y\| .
$$

We call the point $d(x)$ the driver of the flow at $x$. Assigning to the critical points of $h$ the zero vector and to every other point in $\mathbb{R}^{3}$ the unique unit vector of steepest ascent defines a vector field

$$
v: \mathbb{R}^{3} \rightarrow \mathbb{S}^{2} \cup\{0\}
$$

on $\mathbb{R}^{3}$. This vector field is not continuous but nevertheless gives rise to a flow on $\mathbb{R}^{3}$, i.e., a mapping

$$
\phi:[0, \infty) \times \mathbb{R}^{3} \rightarrow \mathbb{R}^{3},
$$

such that at every point $(t, x) \in[0, \infty) \times \mathbb{R}^{3}$ the right derivative

$$
\lim _{t \leftarrow t^{\prime}} \frac{\phi(t, x)-\phi\left(t^{\prime}, x\right)}{t-t^{\prime}}
$$

exists and equals $x$. The flow tells how a point would move if it always followed the steepest ascent of the distance function $h$. The curve that a point $x$ follows is given by $\phi_{x}: \mathbb{R} \rightarrow$ $\mathbb{R}^{3}, t \mapsto \phi(t, x)$ and called the orbit of $x$.

Stable manifolds. Given a critical point $c$ of $h$ the set of all points whose orbit ends in $c$, i.e. the set of all points that flow into $c$, is called the stable manifold of $c$. The collection of all stable manifolds forms a cell complex which is called flow complex. The dimension of each cell in the flow complex is the index of its associated critical point. The cells have a recursive structure, namely, the boundary of the stable manifold of a critical point is made up of stable manifolds of critical points of lower index. Here we summarize the basic facts of the stable manifolds for the different indices of the critical points.

INDEX-0. The stable manifold of an index-0 critical point, i.e., a local minimum, is just the minimum itself.

INDEX-1. The stable manifold of an index-1 critical point, also called a 1-saddle, i.e., the intersection point of a Delaunay edge with its dual Voronoi facet, is the Delaunay edge which in this case is a Gabriel edge.

INDEX-2. The stable manifolds of an index-2 critical point, also called a 2-saddle, is a piecewise linear surface patch. See [12] for details on structure and computation of these patches.

INDEX-3. The stable manifolds of index-3 critical points, i.e. a local maximum, are the bounded regions in the complex built by the stable manifolds of critical points of index 0,1 and 2 .

\section{SEPARATION OF CRITICAL POINTS}

In the following $P$ is always an $\varepsilon$-sample (with $\varepsilon$ to be specified) of a smooth closed surface $\Sigma$ embedded in $\mathbb{R}^{3}$. Also, $h$ is the distance function associated with $P$ and $\phi$ is the flow induced by $P$ following the vector field $v$.

Lemma 3. Let $x$ be a point in $\mathbb{R}^{3} \backslash(\Sigma \cup M)$ with $\mu(x)=\infty$. Then, $x$ is not a critical point of $h$ and the angle between the vectors $\breve{x}-x$ and $v(x)$ is strictly less than $\pi / 2$.

Proof. If $m(x)=\infty$ then $\check{x}$ is at infinity and the hyperplane $H$ tangent to $\Sigma$ at $\hat{x}$ does not have any point from $\Sigma$ on the same side as $\hat{x}$. Therefore, $H$ separates $x$ from $\Sigma$ and in particular from conv $A(x)$. Consequently, $x$ cannot be a critical point of $h$ and for every point $y$ except $\hat{x}$, on the ray from $\hat{x}$ through $x$, the angle between the vectors $v(y)$ and $\hat{y}-y$ is strictly less than $\pi / 2$.

The next lemma states that for $x \in \mathbb{R}^{3} \backslash(\Sigma \cup M)$ with $\mu(x), m(x)<\infty$ there cannot be a critical point of $h$ on the line segment from $\hat{x}$ to $\check{x}$ that is either too close to $\check{x}$ or too close to $\hat{x}$.

LEMmA 4. Let $\varepsilon<1 / 3$ and let $x$ be a point in $\mathbb{R}^{3} \backslash(\Sigma \cup M)$ with $\mu(x)<\infty$. Then $x$ is not a critical point of $h$ and the angle between the vectors $\breve{x}-x$ and $v(x)$ is strictly less than $\frac{\pi}{2}$, provided that $2 \varepsilon \mu(x)<m(x)$ and $\varepsilon^{2} f(\hat{x})<s(x)$.

Proof. For the feature size $f$ at $\hat{x}$ it holds that $f(\hat{x}) \leq$ $\mu(x)$ by the definition of feature size. Thus the closest sample point in $P$ to $\hat{x}$ lies inside a ball centered at $\hat{x}$ with radius at most $\varepsilon f(\hat{x})$. Hence the distance from $x$ to its closest sample point is at most $s(x)+\varepsilon f(\hat{x})$. Consequently, the set $A(x) \subseteq P$, of sample points at minimum distance from $x$, is contained in the ball $B$ centered at $x$ with radius $s(x)+\varepsilon f(\hat{x})$. Let $B^{\prime}$ be the open ball centered at $\check{x}$ with 
radius $\mu(x)$. Since $B^{\prime}$ is empty of any sample points, every point of $A(x)$ is contained in $B \backslash B^{\prime}$. The driver $d(x)$ of the flow induced by $P$ at $x$ is by definition in the convex hull of $A(x)$ and is therefore contained in the convex hull of $B \backslash B^{\prime}$.

Consider the disk $D=H \cap B^{\prime}$ where $H$ is the hyperplane containing $x$ perpendicular to the line through $\hat{x}$ and $\check{x}$. Let $r$ be the radius of this disk. If $s(x)+\varepsilon f(\hat{x})<r$, then $x \notin$ $\operatorname{conv}\left(B \backslash B^{\prime}\right)$. Consequently, $x$ is not a critical point of $h$ and the angle between the vectors $\check{x}-x$ and $v(x)$ is strictly less than $\pi / 2$, see Figure 1 . Thus for the statement of the lemma to hold, it suffices to show that $s(x)+\varepsilon f(\hat{x})<r$. By the Pythagorean theorem

$$
r^{2}=\mu(x)^{2}-m(x)^{2}
$$

and

$$
s(x)+\varepsilon f(\hat{x})=\mu(x)-m(x)+\varepsilon f(\hat{x}) .
$$

Therefore $s(x)+\varepsilon f(\hat{x})<r$ is equivalent to

$$
(\mu(x)-m(x)+\varepsilon f(\hat{x}))^{2}<\mu(x)^{2}-m(x)^{2},
$$

which in turn is equivalent to,

$$
m(x)^{2}-(\mu(x)+\varepsilon f(\hat{x})) m(x)+\varepsilon \mu(x) f(\hat{x})+\frac{\varepsilon^{2}}{2} f(\hat{x})^{2}<0 .
$$

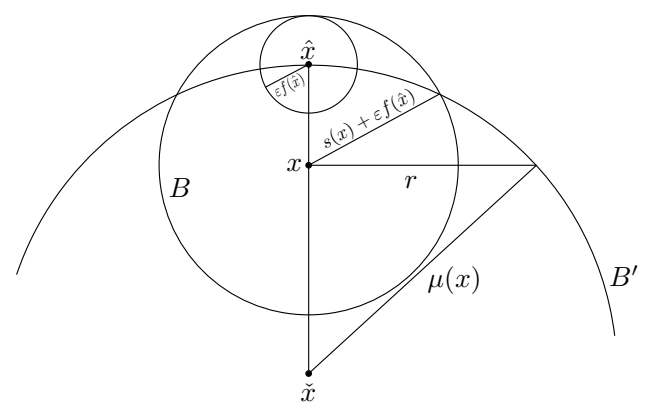

Figure 1. Flow can escape the center of the medial ball only close to surface or close to center.

This inequality holds between the two roots of the quadratic function of $m(x)$ on the left hand side of the inequality, i.e., for

$$
\begin{aligned}
& m(x)> \frac{1}{2}(\mu(x)+\varepsilon f(\hat{x})- \\
&\left.\sqrt{\mu(x)^{2}-\varepsilon^{2} f(\hat{x})^{2}-2 \varepsilon \mu(x) f(\hat{x})}\right) \\
& m(x)<\frac{1}{2}( \mu(x)+\varepsilon f(\hat{x})+ \\
&\left.\sqrt{\mu(x)^{2}-\varepsilon^{2} f(\hat{x})^{2}-2 \varepsilon \mu(x) f(\hat{x})}\right) .
\end{aligned}
$$

Using $f(\hat{x})<\mu(x)$ the lower bound on $m(x)$ can be weakened as follows

$$
\begin{aligned}
m(x)> & \frac{1}{2}(\mu(x)+\varepsilon \mu(x)- \\
& \left.\sqrt{\mu(x)^{2}-\varepsilon^{2} \mu(x)^{2}-2 \varepsilon \mu(x)^{2}}\right) \\
= & \frac{\mu(x)}{2}\left(1+\varepsilon-\sqrt{1-2 \varepsilon-\varepsilon^{2}}\right) .
\end{aligned}
$$

Notice that our assumption that $\varepsilon<1 / 3$ implies

$$
0<1-2 \varepsilon-\varepsilon^{2}<1,
$$

i.e., we have a real lower bound on $m(x)$ and can further weaken this bound by applying the rule $\sqrt{x}>x$ for $x \in(0,1)$ to get

$$
\begin{aligned}
1+\varepsilon-\sqrt{1-2 \varepsilon-\varepsilon^{2}} & <1+\varepsilon-\left(1-2 \varepsilon-\varepsilon^{2}\right) \\
& =3 \varepsilon+\varepsilon^{2}<4 \varepsilon
\end{aligned}
$$

which implies the weaker lower bound $m(x)>2 \varepsilon \mu(x)$. Next we want to show that $s(x)>\varepsilon^{2} f(\hat{x})$ implies the upper bound on $m(x)$, i.e., $s(x)<\varepsilon^{2} f(\hat{x})$ is a weaker form of this upper bound. Assuming $s(x)>\varepsilon^{2} f(\hat{x})$ we get

$$
m(x)=\mu(x)-s(x)<\mu(x)-\varepsilon^{2} f(\hat{x}) .
$$

Thus it is enough to show that

$$
\begin{aligned}
\mu(x)-\varepsilon^{2} f(\hat{x})< & \frac{1}{2}(\mu(x)+\varepsilon f(\hat{x})+ \\
& \left.\sqrt{\mu(x)^{2}-\varepsilon^{2} f(\hat{x})^{2}-2 \varepsilon \mu(x) f(\hat{x})}\right) .
\end{aligned}
$$

The latter inequality is equivalent to

$$
\begin{aligned}
-\frac{3}{4} \mu(x)^{2}+ & \left(\frac{3}{2} \varepsilon-\varepsilon^{2}\right) \mu(x) f(\hat{x})+ \\
& \left(\frac{5}{4} \varepsilon^{2}+\varepsilon^{3}+\varepsilon^{4}\right) f(\hat{x})^{2}<0 .
\end{aligned}
$$

Plugging in $f(\hat{x}) \leq \mu(x)$ we get the stronger inequality

$$
-\frac{3}{4}+\frac{3}{2} \varepsilon+\frac{1}{4} \varepsilon^{2}+\varepsilon^{3}+\varepsilon^{4}<0,
$$

which in turn gives by summarizing

$$
\frac{1}{4} \varepsilon^{2}+\varepsilon^{3}+\varepsilon^{4} \text { into } \frac{9}{4} \varepsilon^{2}
$$

the even stronger inequality

$$
-1+2 \varepsilon+3 \varepsilon^{2}<0
$$

which is satisfied through our assumption that $\varepsilon<1 / 3$. Thus $s(x)>\varepsilon^{2} f(\hat{x})$ implies the upper bound on $m(x)$.

$\delta$-tubular neighborhoods. For a constant $\delta \leq 1$, define $M_{\delta}$ as the union of medial balls, i.e., maximal empty balls, all scaled down by a factor of $\delta$. More formally,

$$
M_{\delta}=\left\{x \in \mathbb{R}^{3} \backslash(\Sigma \cup M): m(x)<\delta \mu(x)\right\} \cup M .
$$

Similarly let

$$
\Sigma_{\delta}=\left\{x \in \mathbb{R}^{3} \backslash(\Sigma \cup M): s(x)<\delta f(\hat{x})\right\} \cup \Sigma .
$$

COROllary 2. Every critical point of the distance function $h$ either belongs to $\Sigma_{\epsilon^{2}}$ or $M_{2 \epsilon}$ provided $\varepsilon<1 / 3$.

Surface and medial axis critical points. Let $\varepsilon<1 / 3$. We call a critical point of $h$ a surface critical point if it is contained in $\Sigma_{\epsilon^{2}}$ and we call it a medial axis critical point if it is contained in $M_{2 \epsilon}$.

\section{ALGORITHMS}

\subsection{Separation of critical points}

Here our goal is to devise an algorithm that can separate the surface critical points from the medial axis critical points. 


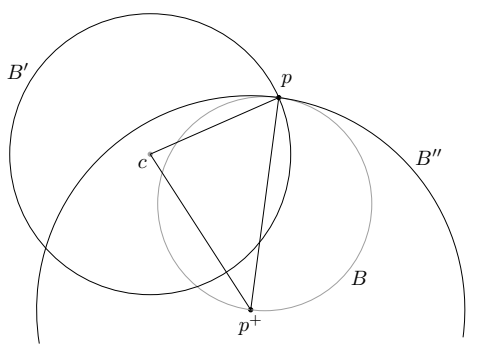

Figure 2. Ball of diameter $\left\|p-p^{+}\right\|$cannot contain any critical associated to $p$.

To prove the correctness of our algorithm we will frequently make use of the following definition.

Associating critical point to samples. A critical point $c$ is said to be associated with a sample point $p \in P$ if $\|p-c\|=$ $h(c)$, i.e., if $c$ is contained in the closed Voronoi cell of $p$.

At first we want to deal with surface critical points. The following two lemmas turn out to be useful to this end.

Lemma 5. For any sample point $p \in P$ the ball $B$ with diameter $\left\|p-p^{+}\right\|$with $p, p^{+} \in \partial B$ does not contain any critical point associated with $p$ in its interior. The analogous statement holds for the negative pole $p^{-}$.

Proof. If $p^{+}$lies at infinity then the ball $B$ becomes a halfspace with normal $\nu_{p}^{+}$. The boundary of this halfspace is a hyperplane that supports the convex hull of $P$. Since any critical point of $h$ must be contained in the convex hull of $P$ it follows that the the interior of $B$, i.e., the open half space, cannot contain any critical point of $h$. Thus we can assume that $p^{+}$is finite. Let $c$ be a critical point associated with $p$. If $c$ is a minimum, then $c=p$ and there is nothing to prove. Otherwise, $|A(c)|>1$. All points in $A(c)$ lie on the boundary of the ball $B^{\prime}$ of radius $\|c-p\|$ centered at $c$. Let $B^{\prime \prime}$ be the open ball of radius $\left\|p-p^{+}\right\|$centered at $p^{+}$. By construction there can be no points of $P$ in $B^{\prime \prime}$. Thus all points of $A(c)$ must belong to $\partial B^{\prime} \backslash B^{\prime \prime}$. On the other hand, for $c$ to be a critical point, it must be in the convex hull of $A(c)$. This happens only if the angle $\angle p c p^{+}$is smaller than $\pi / 2$. The latter condition is in turn identical to $c$ being outside the ball $B$, see Figure 2 . The proof of the analogous statement for $p^{-}$follows the same lines.

LEMMA 6. Let c be a surface critical point associated with sample point $p \in P$. If $\varepsilon<0.1$ then

$$
\|c-p\| \leq \frac{1.1 \varepsilon}{1-1.2 \varepsilon}\left\|p-p^{-}\right\| \leq \frac{1.1 \varepsilon}{1-1.2 \varepsilon}\left\|p-p^{+}\right\| .
$$

Proof. Let $q \in P$ be the closest sample point to $\hat{c}$. By the sampling condition it holds that $\|\hat{c}-q\| \leq \varepsilon f(\hat{c})$. Using the triangle inequality we obtain

$$
\begin{aligned}
\|c-p\| & \leq\|c-q\| \leq s(c)+\|\hat{c}-q\| \\
& \leq \varepsilon^{2} f(\hat{c})+\varepsilon f(\hat{c})=\varepsilon(1+\varepsilon) f(\hat{c}) .
\end{aligned}
$$

Since the local feature size $f$ is 1-Lipschitz we can write

$$
\begin{aligned}
f(\hat{c}) & \leq f(p)+\|\hat{c}-p\| \\
& \leq f(p)+s(c)+\|c-p\| \\
& <f(p)+\varepsilon^{2} f(\hat{c})+\varepsilon(1+\varepsilon) f(\hat{c}) \\
& =f(p)+\varepsilon(1+2 \varepsilon) f(\hat{c}) \\
& <f(p)+1.2 \varepsilon f(\hat{c}) .
\end{aligned}
$$

Rearranging, we obtain

$$
f(\hat{c})<\frac{1}{1-1.2 \varepsilon} f(p) .
$$

Combining (1) and (2) we finally get

$$
\begin{aligned}
\|c-p\| & \leq \varepsilon(1+\varepsilon) f(\hat{c})<\frac{\varepsilon(1+\varepsilon)}{1-1.2 \varepsilon} f(p) \\
& <\frac{1.1 \varepsilon}{1-1.2 \varepsilon} f(p) \leq \frac{1.1 \varepsilon}{1-1.2 \varepsilon}\left\|p-p^{-}\right\| \\
& \leq \frac{1.1 \varepsilon}{1-1.2 \varepsilon}\left\|p-p^{+}\right\| .
\end{aligned}
$$

From Lemmas 5 and 6 we derive the following corollary.

Corollary 3. Let c be a surface critical point associated with sample point $p \in P$. Let $\varepsilon<0.1$. Then the acute angles between the vector $c-p$ and each of the vectors $\nu_{p}^{+}$and $\nu_{p}^{-}$ is at least 75.5 degrees.

Proof. By Lemma 5 the ball of diameter $\left\|p^{-}-p\right\|$ with $p^{-}$and $p$ on its boundary and the ball of diameter $\left\|p^{+}-p\right\|$ with $p^{+}$and $p$ on its boundary both contain no critical points. Thus we get from using Lemma 6 and Thales theorem an upper bound of

$$
\frac{1.1 \epsilon}{1-1.2 \epsilon}
$$

for the cosine of the angle between the vector $c-p$ and each of the vectors $\nu_{p}^{+}$and $\nu_{p}^{-}$. Thus for $\varepsilon<0.1$, the resulting angles are at least 82.5 degrees. But note that so far we can not conclude that these angles are acute. The angle between the vectors $\nu_{p}^{+}$and $\nu_{p}^{-}$is at least

$$
\pi-4 \arcsin \frac{\varepsilon}{1-\varepsilon}
$$

by Corollary 1 . Since $\varepsilon<0.1$ this angle is at least 173 degrees. Thus the angles between $c-p$ and each of $\nu_{p}^{+}$and $\nu_{p}^{-}$ are at most

$$
82.5+(360-165-173)=104.5 \text { degrees. }
$$

From this, the acute angles between $c-p$ and each of $\nu_{p}^{+}$ and $\nu_{p}^{-}$is at least $180-104.5=75.5$ degrees.

Next we deal with medial axis critical points.

LEMMA 7. Let $c$ be a medial axis critical point associated with $p \in P$. If $\varepsilon<1 / 3$ then $\|p-c\| \geq(1-2 \varepsilon) f(p)$.

Proof. We have $s(c) \leq\|c-p\|$ since $s(c)$ is the distance from $c$ to $\Sigma$ and we have $m(c), \mu(c)<\infty$ by Lemma 3. The definition of a medial axis critical point states

$$
m(c) \leq 2 \varepsilon \mu(c)=2 \varepsilon(m(c)+s(c)) .
$$


Combining these inequalities we obtain

$$
m(c) \leq \frac{2 \varepsilon}{1-2 \varepsilon} s(c) \leq \frac{2 \varepsilon}{1-2 \varepsilon}\|c-p\|,
$$

which gives

$$
\begin{aligned}
f(p) & \leq\|\check{c}-p\| \leq m(c)+\|c-p\| \\
& \leq\left(\frac{2 \varepsilon}{1-2 \varepsilon}+1\right)\|c-p\|=\frac{1}{1-2 \epsilon}\|c-p\|
\end{aligned}
$$

and thus $\|p-c\| \geq(1-2 \varepsilon) f(p)$.

Corollary 4. Let $c$ be a medial axis critical point associated with $p \in P$. If $\varepsilon<0.1$ then the acute angle between the vector $c-p$ and each of the vectors $\nu_{p}^{-}$and $\nu_{p}^{+}$is at most 28 degrees.

Proof. Using Lemma 7 we can plug in $1-2 \varepsilon$ for $\delta$ in Lemma 2 to obtain that the angle between $x-p$ and each of $\nu_{p}^{+}$or $\nu_{p}^{-}$is at most

$$
3 \arcsin \frac{\varepsilon}{1-\varepsilon}+\arcsin \frac{\varepsilon}{(1-2 \varepsilon)(1-\varepsilon)} .
$$

The statement of the Lemma now follows from $\varepsilon<0.1$.

Corollary 3 and Corollary 4 show that for a critical point $c$ associated to a sample $p$, the angle between $c-p$ and a pole of $p$ falls into one of two disjoint ranges 0 to 45 degrees or 75.5 to 90 degrees, depending on whether $c$ is a surface or a medial axis critical point. Thus by looking at this angle, we can distinguish between these two types as follows: for any sample point $p$ and any critical point $c$ associated with $p$, we measure the angle $\alpha_{c}=\angle\left(\nu_{p}, c-p\right)$, where $\nu_{p}$ is either of the two pole vectors at $p$. If $\alpha_{c}<\pi / 4$ or $\alpha_{c}>3 \pi / 4$, then $c$ is a medial axis critical point and otherwise a surface one.

\subsection{Reconstruction}

The algorithmic classification of the critical points of $h$ as either surface or medial axis critical points suggests the following algorithm to reconstruct $\Sigma$ from $P$. By $\operatorname{Separate}(P, C)$ we refer the to the algorithm described above that partitions the critical points into surface and medial axis ones.

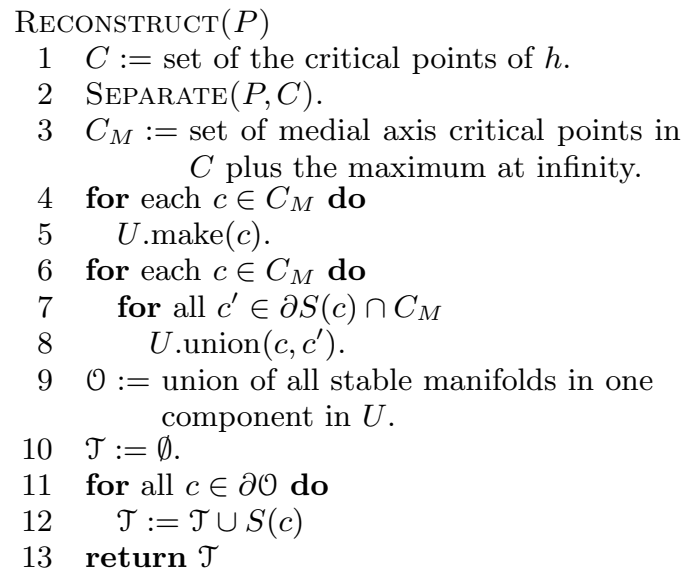

The algorithm RECONSTRUCT builds on a Union-Find data structure $U$ on the set of medial axis critical points. In line
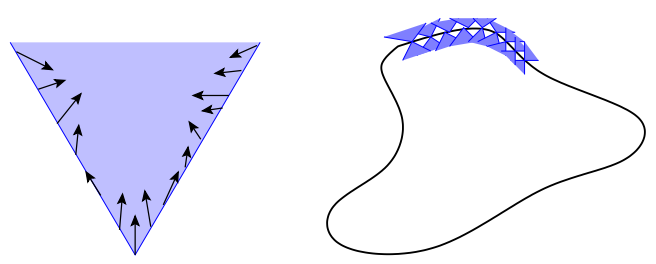

Figure 3. Sink cones

5, $U$.make $(c)$ adds a singleton set $\{c\}$ to $U$ and in line 9, $U$.union $\left(c, c^{\prime}\right)$ combines the sets containing $c$ and $c^{\prime}$ into a single set. The Union-Find data structure is used to find all connected components of stable manifolds $S(c)$ of medial axis critical points $c \in C_{M}$. In the end the boundary of one arbitrary component is output. Notice that this boundary is made of stable manifolds of surface critical points.

\section{RECONSTRUCTION PROPERTIES}

In this section, we give geometric and topological guarantees for the output of the algorithm RECONSTRUCT under $\varepsilon$-sampling. We summarize the results in the following Theorem.

TheOREM 1. For any $0<\rho<1$ there exists $\varepsilon_{0}$ such that given an $(\varepsilon, \delta)$-sample $P$ from a smooth closed surface $\Sigma$, where $\varepsilon<\varepsilon_{0}$ and $\delta / \varepsilon=\rho$, the algorithm RECONSTRUCT outputs a subcomplex $\mathcal{T}$ of the flow complex with the following properties:

(i) $\mathcal{T}$ is contained in the tubular neighborhood $\Sigma_{12 \varepsilon^{2}}$.

(ii) The normal of a triangle pqr in $\mathcal{T}$, where $p \in P$, forms an angle of $O(\varepsilon)$ with the normal of $\Sigma$ at $p$.

(iii) $\mathcal{T}$ is homeomorphic (in fact, isotopic) to $\Sigma$.

In particular these claims hold for $\rho \geq 3 / 4, \varepsilon_{0} \leq 0.01$.

\subsection{Closeness}

To analyze local geometry of the flow near the surface, we place at sample points $p \in P$ cones that open along inner and outer normal directions at $p$. We show that, under certain conditions, such cones are sinks, i.e., on their surfaces the flow is either tangential or points to the inside of the cones. Of course this is not shown to hold everywhere on the surface of such cones - certainly, we could not make such a claim for far away points - but only points close enough to surface. However, by overlapping together these close-reaching cones (see Figure 3), we obtain inner and outer envelopes that enclose the surface and are in a sense "one-way" for the flow. This means that flow cannot escape from these envelopes leading to properties of the flow complex important to the analysis of the output of our algorithm.

Sink Cones. For a point $p$, and a direction vector $n$, let $C=\operatorname{cone}(p, n, \theta, r)$ be the cone-patch consisting of points $x$ for which $\|x-p\| \leq r$ and $\angle(n, x-p)=\theta$. We call $\theta$, and $r$, the angle and the reach of $C$, respectively. A cone-patch is essentially part of the surface of an infinite cone. With a slight abuse of notation we refer to a cone-patch also as a 


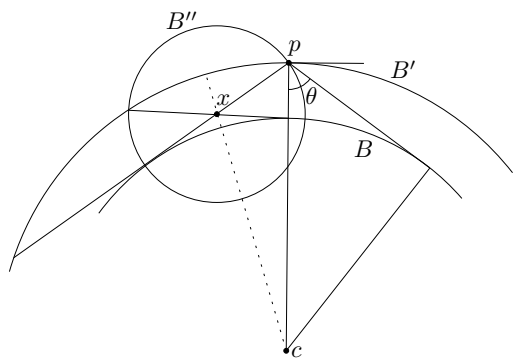

Figure 4. Sink cone for a sample point $p$

cone. The boundary of $C$ consists of points $x \in C$ for which $\|x-p\|=r$. We say that $C$ is a sink if at every point of the relative interior of $C$ (thus excluding the boundary of $C$ ) the flow is either tangential or directed toward the interior of the convex hull of $C$.

Lemma 8. For any point $p \in P, C_{p}^{+}=\operatorname{cone}\left(p, n_{p}^{+}, \theta, r\right)$ and $C_{p}^{-}=\operatorname{cone}\left(p, n_{p}^{-}, \theta, r\right)$ are sinks for any $0<\theta<\frac{\pi}{2}$ and $r=f(p) \cos \theta$. Furthermore, the interior of the convex hulls of $C_{p}^{+}$and $C_{p}^{-}$do not contain any critical points.

Proof. We only prove that $C_{p}^{+}$is a sink. The proof for $C_{p}^{-}$is similar. Let $c$ be the point on the ray in direction $n_{p}^{+}$at distance $f(p)$ from $p$. Let $B^{\prime}$ be the ball with radius $\|p-c\|$ centered at $c$. Note that $B^{\prime}$ cannot contain any points from $\Sigma$ in its interior since it is contained in a medial ball. Let $B$ be the ball centered at $c$ with radius $\left(1-\varepsilon^{2}\right) f(p)$. Then $C_{p}^{+}$ is the cone tangent to $B$ with apex $p$ (see Figure 4 ). The set $A(x)$ of closest sample points to $x$ are inside the ball $B^{\prime \prime}$ of radius $\|x-p\|$ centered at $x$ but outside $B^{\prime}$. Let $H$ be the hyperplane tangent to $C_{p}^{+}$at $x$. Since $r=f(p) \sin \theta$ we have that $\operatorname{conv}\left(B^{\prime \prime} \backslash B^{\prime}\right)$ is entirely on the opposite side of $H$ with respect to $B$ and therefore, $v(x)$ points toward the interior of $C_{p}^{+}$. Thus $C_{p}^{+}$is a sink and no point in the relative interior of $C_{p}^{+}$is a critical point. Every point in the interior of the convex hull of $C_{p}^{+}$is on a cone cone $\left(p, n_{p}^{+}, \theta^{\prime}, r\right)$ for some $\theta^{\prime}<\theta$ or in the relative interior of the line segment $p c$. As we have seen the points on the cones cannot be critical. But neither can be any point $y$ in the relative interior of the line segment $p c$ since for it $A(y)=\{p\}$ and $y \neq p$.

Fixing cone angles. Notice that the above lemma puts at every sample point two sink cones with the same apex, angle, reach, and axis but in opposite direction. For the rest of the paper, we shall consider only such cones with a fixed cone angle that depends only on the density of sampling. Indeed, we fix $\theta=\theta(\varepsilon)=\frac{\pi}{2}-5 \varepsilon$ and we respectively denote the outer and inner cones at a vertex $p$ by $C_{p}^{-}=$ $\operatorname{cone}\left(p, n_{p}^{-}, \theta, f(p) \cos \theta\right)$ and $C_{p}^{+}=\operatorname{cone}\left(p, n_{p}^{+}, \theta, f(p) \cos \theta\right)$. We also denote by $C_{p}$ the union of the two cones $C_{p}^{+}$and $C_{p}^{-}$.

Lemma 9. For any point $x \in V_{p} \cap \Sigma$, the ray shot in direction $n_{x}^{+}\left(n_{x}^{-}\right)$hits $C_{p}^{+}\left(C_{p}^{-}\right)$provided that $\varepsilon \leq 0.01$.

Proof. Let $\beta$ be the angle between $n_{x}^{+}$and $n_{p}^{+}$. Since $x \in V_{p} \cap \Sigma$, we have $\|x-p\| \leq \varepsilon f(x)$ and therefore $\beta \leq$ $\frac{\varepsilon}{1-3 \varepsilon}<\theta$. Therefore, the ray shot from $x$ in direction of $n_{x}^{+}$hits cone $\left(p, n_{p}^{+}, \theta, \infty\right)$, the infinite extension of $C_{p}^{+}$, at some point $x^{\prime}$. Let $\eta=\left\|x^{\prime}-p\right\| / f(p)$. If $\eta \leq \cos \theta$, then $x^{\prime} \in C_{p}^{+}$and there is nothing to prove. Otherwise $\left\|x^{\prime}-p\right\|>$ $f(p) \cos \theta$. It can be easily observed that the closest point to $p$ on the line through $x^{\prime}$ and $x$ is at distance no less than

$$
\left\|x^{\prime}-p\right\| \sin (\theta-\beta)>\cos \theta \sin (\theta-\beta) f(p)
$$

from $p$. On the other hand, since $x \in V_{p} \cap \Sigma,\|x-p\| \leq$ $\frac{\varepsilon}{1-\varepsilon} f(p)$. This implies

$$
\begin{aligned}
\frac{\varepsilon}{1-\varepsilon} f(p) & \geq\|x-p\| \\
& >\cos \theta \sin (\theta-\beta) f(p) \\
& =\sin (5 \varepsilon) \cos (5 \varepsilon+\beta) f(p) \\
& \geq \sin (5 \varepsilon) \cos \left(5 \varepsilon+\frac{\varepsilon}{1-3 \varepsilon}\right) f(p)
\end{aligned}
$$

which is a contradiction for $\varepsilon \leq 0.01$.

Lemma 10. Let $x$ be a point on $C_{p}$ for which $\hat{x} \in V_{p}$. Then $\|x-\hat{x}\| \leq \frac{2 \varepsilon \cos \theta}{1-2 \cos \theta} \cdot f(\hat{x}) \leq 12 \varepsilon^{2} f(\hat{x})$ when $\varepsilon \leq 0.01$.

Proof. Let $\eta=\|x-p\| / f(\hat{x})$. Without loss of generality assume that $x \in C_{p}^{+}$(the proof for the case where $x \in C_{p}^{-}$ is similar). Shoot a ray from $x$, parallel to $n_{p}^{-}$until it hits $C_{p}^{-}$at a point $x^{\prime}$. Since each of $C_{p}^{+}$and $C_{p}^{-}$is completely contained in its corresponding medial ball tangent to $\Sigma$ at $p$, the line segment $x x^{\prime}$ intersects $\Sigma$. Therefore

$$
\|x-\hat{x}\| \leq\left\|x-x^{\prime}\right\|=2\|x-p\| \cos \theta=2 \eta f(\hat{x}) \cos \theta .
$$

On the other hand, by triangle inequality,

$$
\begin{aligned}
\|\hat{x}-p\| & \geq\|p-x\|-\|x-\hat{x}\| \\
& \geq \eta f(\hat{x})-2 \eta f(\hat{x}) \cos \theta \\
& =\eta(1-2 \cos \theta) f(\hat{x}) .
\end{aligned}
$$

However, since $\hat{x} \in V_{p}$, by sampling condition $\|\hat{x}-p\| \leq$ $\varepsilon f(\hat{x})$. Thus we must have $\eta \leq \varepsilon /(1-2 \cos \theta)$. Plugging this bound for $\eta$ into (3) we get

$$
\|x-\hat{x}\| \leq \frac{2 \varepsilon \cos \theta}{1-2 \cos \theta} \cdot f(\hat{x}) \leq 12 \varepsilon^{2} f(\hat{x}),
$$

for $\varepsilon \leq 0.01$ and $\theta=\pi / 2-5 \varepsilon$.

The following lemma will be used to show that for appropriate choice of $\theta$, and under dense enough sampling, the sample point cones on each side of $\Sigma$ patch up to construct envelopes from which the flow cannot escape.

Lemma 11. For a sample point $p$, let $x$ be a point on the boundary of $C_{p}^{+}\left(C_{p}^{-}\right)$, i.e. $\|x-p\|=f(p) \cos \theta$. Let $q$ be a closest sample point to $\hat{x}$. Then the segment $x \hat{x}$ intersects the relative interior of $C_{q}^{+}\left(C_{q}^{-}\right)$provided that $\varepsilon \leq 0.01$.

Proof. We prove the lemma for the case of $x$ on the boundary of $C_{p}^{+}$(the proof of $C_{p}^{-}$is analogous). Consider a ball $B$ of radius $f(p)$ tangent to $\Sigma$ at $p$ and in the exterior of $\Sigma$. Let $c$ be the center of $B$. It can be easily observed that $\|c-x\|=f(p) \sin \theta$. Therefore, the distance from $x$ to boundary of $B$ is $(1-\sin \theta) f(p)$. Since the interior of $B$ contains no point of $\Sigma$, we get

$$
\|x-\hat{x}\| \geq(1-\sin \theta) f(p)
$$

By triangle inequality we have

$$
\mid \hat{x}-p\|\leq\| \hat{x}-x\|+\| x-p\|\leq 2\| x-p \|=2 f(p) \cos \theta .
$$


From Lipschitzness of local feature size we get

$$
f(p) \geq f(\hat{x})-\|\hat{x}-p\| \geq f(\hat{x})-2 f(p) \cos \theta .
$$

Therefore $f(p) \geq f(x) /(1+2 \cos \theta)$. Combining this with (4) we get

$$
\|x-\hat{x}\| \geq \frac{1-\sin \theta}{1+2 \cos \theta} \cdot f(\hat{x}) .
$$

On the other hand, by Lemma 9 , since $q$ is the closest sample point to $\hat{x}$, the ray shot in outward normal direction at $\hat{x}$ hits $C_{q}^{+}$in some point $y$. Therefore, by Lemma 10 ,

$$
\|y-\hat{x}\| \leq \frac{2 \varepsilon \cos \theta}{1-2 \cos \theta} f(\hat{x}) .
$$

The proof of the lemma becomes complete by verifying that for $\varepsilon \leq 0.01$ the following inequality holds:

$$
\frac{2 \varepsilon \cos \theta}{1-2 \cos \theta}<\frac{1-\sin \theta}{1+2 \cos \theta}
$$

Cone envelopes. Let $C^{+}=\bigcup_{p \in P} C_{p}^{+}$and let $\Sigma^{+}$be the set of points $x \in C^{+}$that are closest points in $C^{+}$to $\hat{x}$ in the normal direction $n_{\hat{x}}^{+}$. We call $\Sigma^{+}$the outer cone envelope of $\Sigma$. The inner cone envelope $\Sigma^{-}$is defined analogously.

LEMMA 12. The surface $\Sigma$ is homeomorphic to both $\Sigma^{-}$ and $\Sigma^{+}$. Both cone envelopes divide $\mathbb{R}^{3}$ into a bounded and an unbounded component. The bounded component of the inner cone envelope and the unbounded component of the outer cone envelope are closed under the flow $\phi$.

Proof. We only the discuss the outer cone envelope. The proof for the inner cone envelope follows the same lines. Let $\pi^{+}$be the mapping that maps any point $x \in \Sigma$ to its closest point $x^{\prime}$ on one of the cones $C_{p}^{+}$in the normal direction $n_{x}^{+}$. By Lemma 9 the map is well-defined at every point $x \in \Sigma$ because the ray shot in normal direction $n_{x}^{+}$hits the outer cone $C_{p}^{+}$placed at closest sample point $p$ to $x$. By definition $\pi^{+}(x) \in \Sigma^{+}$for every $x \in \Sigma$ and by construction $\pi^{+}(\Sigma)=\Sigma^{+}$. That is, $\pi^{+}: \Sigma \rightarrow \Sigma^{+}$is onto. Since the map $x \mapsto n_{x}^{+}$is continuous on $\Sigma$ the mapping $\pi^{+}$is also continuous except at any points $x$ that is mapped to a boundary points of some cone $C_{p}^{+}$. But by Lemma 11, no point of $\Sigma$ is mapped by $\pi^{+}$into the boundary of any cone $C_{p}^{+}$since there is always a closer cone in the way. Therefore, the mapping $\pi^{+}$is continuous everywhere. Finally, $\pi^{+}$is one-to-one, because by construction the vector $\pi^{+}(x)-x$ is normal to $\Sigma$ at $x$, which means that $x$ is a closest point to $\pi^{+}(x)$ on $\Sigma$, which is unique since $\pi^{+}(x)$ cannot be a point on the medial axis by Lemma 10 .

Since we assumed that $\Sigma$ is a manifold without boundary, so is $\Sigma^{+}$. Thus $\Sigma^{+}$divides $\mathbb{R}^{3}$ into a bounded and an unbounded component. By Lemma 8 the bounded component has to be closed under the flow $\phi$.

Cone neighborhood. We call the closed volume sandwiched between $\Sigma^{-}$and $\Sigma^{+}$the cone neighborhood of $\Sigma$ and denote it by $\tilde{\Sigma}$.

THEOREM 2. The output of the algorithm RECONSTRUCT lies in $\Sigma_{12 \varepsilon^{2}}$.
Proof. By Lemmas 8 and 12, the stable manifold $S(c)$ of any surface critical point $c$ has to be contained in $\tilde{\Sigma}$. Thus the output of ReCOnstruct completely lies in $\tilde{\Sigma}$. By Lemma $10, \Sigma^{+}$and $\Sigma^{-}$are contained in $\Sigma_{12 \varepsilon^{2}}$. This implies that $\tilde{\Sigma}$ is also contained in $\Sigma_{12 \varepsilon^{2}}$.

Corollary 5. For every $x \in \tilde{\Sigma},\|x-p\| \leq 1.6 \varepsilon f(p)$ for every $p \in A(x)$, provided that $\varepsilon \leq 0.01$.

Proof. Let $\xi=12 \varepsilon^{2}$. Since $\tilde{\Sigma} \subset \Sigma_{\xi}$, we have $x \in \Sigma_{\xi}$, which means $\|x-\hat{x}\| \leq \xi f(\hat{x})$. On the other hand by definition of $\varepsilon$-sampling, $\|\hat{x}-q\| \leq \varepsilon f(\hat{x})$, where $q$ is a closest sample point to $\hat{x}$. By triangle inequality

$$
\|x-p\| \leq\|x-q\| \leq\|x-\hat{x}\|+\|\hat{x}-q\| \leq(\varepsilon+\xi) f(\hat{x}) .
$$

Since local feature size is Lipschitz

$\|p-\hat{x}\| \leq\|x-p\|+\|p-\hat{x}\| \leq\|q-x\|+\xi f(\hat{x}) \leq(\varepsilon+2 \xi) f(\hat{x})$,

and from this

$$
f(p) \geq f(\hat{x})-\|\hat{x}-p\| \geq f(\hat{x})-(\varepsilon+2 \xi) f(\hat{x}) .
$$

Combining (5) and (6) we get

$$
\|x-p\| \leq \frac{\varepsilon+2 \xi}{1-\varepsilon-2 \xi} f(p) \leq 1.6 f(p),
$$

for $\varepsilon \leq 0.01$.

\subsection{Normal}

The output $\mathcal{T}$ produced by the algorithm RECONSTRUCT consists of stable manifolds of index-2 saddle points that lie in a small tubular neighborhood of the surface. We refer to these stable manifolds as surface patches. We want to show that under $(\varepsilon, \delta)$-sampling, with a fixed $\rho=\delta / \varepsilon$, the normal of triangles in these surface patches is within $O(\varepsilon)$ from the normal to surface at a nearby point, for sufficiently small $\varepsilon$. We use the following two lemmas from [3].

Lemma 13. For any two points $p, q \in \Sigma$, the angle between segment $p q$ and either of $n_{p}^{+}$and $n_{p}^{-}$is greater than $\frac{\pi}{2}-\arcsin \frac{\|p-q\|}{2 f(p)}$.

Lemma 14. For points $p, q, r \in \Sigma$, let $p$ be a vertex of the triangle pqr with the largest angle and let $r$ be its circumradius. If $r=\lambda f(p)$, then the angle between the normal to $p q r$ and the normal to surface at $p$ is at most $\beta(\lambda)$ where

$$
\left.\beta(\lambda)=\arcsin (\lambda)+\arcsin \left(\frac{2}{\sqrt{3}} \sin (2 \arcsin \lambda)\right)\right) \leq 5 \lambda,
$$

for $\lambda \leq \frac{1}{2}$.

The stable manifold $S(c)$ of every 2 -saddle is a piece-wise linear surface made of a finite number of triangles, which we call patch triangles. Each patch triangle $t$ has exactly one vertex in $P$. Note that for every point $x$ in a patch triangle $t$, the vertex of $t$ that belongs to $P$ is a closest sample point to $x$ (refer to [12] for details on the structure of stable manifolds of critical points). If $x$ is on the boundary of $t$, it can have more than one closest sample point as it belongs to more than one patch triangle. The following lemma shows that under tight sampling, each patch triangle must have a normal close to surface normal at its vertex in $P$. 


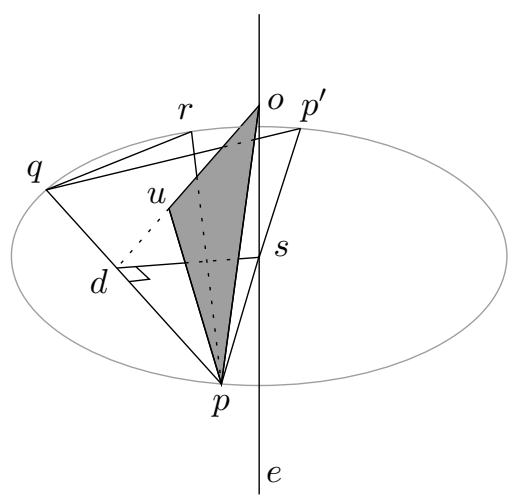

Figure 5. A generic patch triangle on the stable manifold of a surface 2-saddle

Lemma 15. For any $0<\rho<1$, there exists $\varepsilon_{0}$ such that if $P$ is an $\varepsilon$-sample of $\Sigma$ with $\varepsilon \leq \varepsilon_{0}$, then for any $x \in$ $S(c)$, the stable manifold of a surface 2-saddle c, the acute angle between $n_{p}$, where $p$ is a closest sample point to $x$, and $n_{t}$, the normal direction of the patch triangle $t \subset S(c)$ that contains $x$ and has $p$ as a vertex, is at most

$$
\arcsin \left(\frac{\sin (5 \varepsilon)}{2 \sin \left(\frac{1}{2} \arcsin (\rho / 3.2)\right)}\right)=O(\varepsilon / \rho) .
$$

Proof. We ensure in advance for the proof of this lemma that $\varepsilon_{0}$ is at most 0.01 . Let $P$ be an $\varepsilon$-sample of $\Sigma$ for $\varepsilon \leq \varepsilon_{0}$. By Corollary $5,\|x-p\| \leq 1.6 \varepsilon f(p)$ for every closest sample point $p$ to $x$. Every point $x$ on $S(c)$ is on a patch triangle $t=p o u$ of $S(c)$ with the following structure (see Figure 5): $t$ has exactly one vertex $p$ in $P$. The edge $u o$ of $t$ opposite to $p$ is on the Voronoi facet dual to a Delaunay edge $p q$ and ends on the dual Voronoi edge $e$ of a Delaunay triangle $p q r$ in which $\|r-p\|>\|q-p\|$. The mid-point $d$ of $p q$ is the driver of the points on $u o$. Furthermore, the line containing $e$ does not intersect the triangle pqr except when $o$ is the critical point $c$ in which case the patch triangle $t$ is a subset of the Delaunay triangle $t_{c}$ containing $c$. We postpone the study of this special case for later. Let $s$ be the circumcenter of $p q r$ and let $p^{\prime}$ be a point on the circumcircle of $p q r$ opposite to $p$ with respect to $s$. Then $\angle p^{\prime} q p=\pi / 2$ and that $\|d-s\|=$ $\frac{1}{2}\left\|q-p^{\prime}\right\|$. Furthermore, $\left\|q-p^{\prime}\right\|>\|q-r\|$. Therefore we get $\|d-s\| \geq \frac{1}{2} \delta f(r)$. On the other hand, since $r \in A(o)$, by Corollary $5,\|s-p\|=\|s-r\|<\|r-o\| \leq 1.6 \varepsilon f(r)$. Combining these we get for the angle $\alpha=\angle q p s$ :

$$
\sin \alpha \geq \frac{\|d-s\|}{\|p-s\|} \geq \frac{\delta}{3.2 \varepsilon}=\frac{\rho}{3.2} .
$$

On the other hand, $\frac{\pi}{2}>\angle q p o>\angle q p s \geq \alpha$. Also, $o \in S(c)$ and $S(c)$ is contained in $\tilde{\Sigma}$ and therefore, po makes an angle of at least $\theta=\frac{\pi}{2}-5 \varepsilon$ with $n_{p}$. Moreover, $\|p-q\| \leq 3.2 \varepsilon f(p)$ and therefore by Lemma 13 makes an angle of at least $\frac{\pi}{2}-$ $1.6 \varepsilon>\theta$ with $n_{p}$. Putting everything together, the three points $p, q$, and $o$, make a triangle $t^{\prime}=p q o$ with an angle of $\alpha$ at vertex $p$ satisfying $\arcsin (\rho / 3.2)<\alpha<\frac{\pi}{2}$, and with both of the edges incident to $p$ making an angle of at least $\theta$ with $n_{p}$. It can be shown through elementary calculations that under this conditions, $n_{t^{\prime}}$, the normal to $t^{\prime}$, and $n_{p}$, make an angle of at most

$$
\arcsin \left(\frac{\cos \theta}{2 \sin (\alpha / 2)}\right) \text {. }
$$

In the special case that $o$ coincides with $c, e$ intersects its dual triangle $p q r$ at $c$ and the patch triangle $t$ under question becomes a subset of the Delaunay triangle $t_{c}=p q r$. Since $\|p-o\| \leq 1.6 \varepsilon f(p)$ is the circumradius of $t_{c}$, by Lemma 14, the angle between normal to $p q r$ and normal to $\Sigma$ at $p$ is at most $\frac{1.6 \varepsilon}{1-3 \cdot 1.6 \varepsilon}$ which for $\varepsilon \leq 0.01$ is a tighter bound than the one the statement of lemma claims.

Corollary 6. For $\varepsilon \leq 0.01$ and $\rho \geq 3 / 4$, we have for every point $x$ on the stable manifold of a surface 2-saddle $c$ that the acute angle between normal $n_{t}$ to any patch triangle $t$ of $S(c)$ that contains $x$, and the normal $n_{\hat{x}}$ to $\Sigma$ at $\hat{x}$ is at most 14 degrees.

Proof. Plugging $\varepsilon \leq 0.01$ and $\rho \geq 1 / 2$ in Lemma 15, gives an upper bound of 13 degrees for the angle between $n_{t}$ and $n_{p}$, where $n_{p}$ is the normal to $\Sigma$ at a closest sample point $p$ to $x$. Since by Theorem $2, S(c)$ is contained in $\Sigma_{12 \varepsilon^{2}}$, we have $\|x-\hat{x}\| \leq 12 \varepsilon^{2} f(\hat{x})$. Let $q$ be a closest sample point to $\hat{x}$. By sampling condition, $\|\hat{x}-q\| \leq \varepsilon f(\hat{x})$ and therefore

$$
\|\hat{x}-p\| \leq\|\hat{x}-x\|+\|x-p\| .
$$

On the other hand $\|x-p\| \leq\|x-\hat{x}\|+\|\hat{x}-q\|$. Therefore we get

$\|\hat{x}-p\| \leq 2\|x-\hat{x}\|+\|\hat{x}-q\| \leq\left(24 \varepsilon^{2}+\varepsilon\right) f(\hat{x}) \leq 1.5 \varepsilon f(\hat{x})$, for $\varepsilon \leq 0.01$. Therefore by Lemma 1 the angle between $n_{p}^{+}$ and $n_{\hat{x}}^{+}$is at most $1.5 \varepsilon /(1-3 \cdot 1.5 \varepsilon) \leq 1^{\circ}$.

The following Proposition is a direct consequence of the structure of the stable manifolds of surface 2-saddles [12].

Proposition 1. If $t_{1}$ and $t_{2}$ are patch triangles of $S(c)$ for a surface 2-saddle $c$ such that $t_{1}$ and $t_{2}$ have one edge in common, then the dihedral angle between $t_{1}$ and $t_{2}$ is no less than $\pi / 2$.

Lemma 16. Let c be a surface 2-saddle. Suppose we orient the patch triangles in $S(c)$ arbitrarily but consistently so that for any patch triangle $t, n_{t}^{+}$and $n_{t}^{-}$are respectively the outer and inner normal directions on $t$ with respect to the applied orientation. Then, under the assumptions of Corollary 6 exactly one of the following cases holds.

1. $\angle\left(n_{t}^{+}, n_{\hat{x}}^{+}\right) \leq 14^{\circ}$, for every patch triangle $t$ of $S(c)$ and for every $x \in t$.

2. $\angle\left(n_{t}^{+}, n_{\hat{x}}^{-}\right) \leq 14^{\circ}$, for every patch triangle $t$ of $S(c)$ and for every $x \in t$.

Proof. First notice that as was shown in the proof of Corollary 6 , for any point $x \in t$, where $t$ is a patch triangle of $S(c), \angle\left(n_{\hat{x}}^{+}, n_{p}^{+}\right) \leq 1^{\circ}$, where $p$ is the vertex of $t$ that is a sample point. Thus, if for the arbitrary orientation of $t$ and for a point $x \in t, \angle\left(n_{t}^{+}, n_{\hat{x}}^{+}\right)=\alpha$, the same holds for every other point $y$ in $t$, modulo changing $\alpha$ by 1 degree.

Let $t_{c}$ be the Delaunay triangle that contains $c$. All patche triangles $t \subset t_{c}$ of $S(c)$, have the same $n_{t}^{+}$which agrees with one of the two normal direction to $t_{c}$. By Corollary 6 , the 
normal direction of $t_{c}$ makes an angle of at most $14^{\circ}$ with either $n_{\hat{c}}^{+}$or $n_{\hat{c}}^{-}$. Assume without loss of generality that the first case holds, i.e. $\angle\left(n_{t}^{+}, n_{\hat{c}}^{+}\right) \leq 14^{\circ}$. We show now that this will imply that that for every patch triangle $t$ of $S(c)$ and every $x \in t, \angle\left(n_{t}^{+}, n_{\hat{x}}^{+}\right) \leq 14^{\circ}$. We prove this by extending the result for the triangles we already have this property for to their neighboring patch triangles. Thus, assume $t$ and $t^{\prime}$ are two patch triangles with an edge $e$ in common. Let $z$ be a point on $e$. Since $t$ and $t^{\prime}$ are oriented consistently, the dihedral angle between $t$ and $t^{\prime}$ is $\pi-\angle\left(n_{t}^{+}, n_{t^{\prime}}^{+}\right)$. By Proposition 1 this angle is at least $\pi / 2$ and therefore $\angle\left(n_{t}^{+}, n_{t^{\prime}}^{+}\right) \leq \pi / 2$. Therefore using triangle inequality for angles we get $\angle\left(n_{\hat{z}}^{+}, n_{t^{\prime}}^{+}\right) \leq \angle\left(n_{t}^{+}, n_{\hat{z}}^{+}\right)+\angle\left(n_{t}^{+}, n_{t^{\prime}}^{+}\right) \leq 104^{\circ}$. But by Corollary $6, \angle\left(n_{\tilde{z}}^{+}, n_{t^{\prime}}^{+}\right)$is either less than $14^{\circ}$ or more than $180^{\circ}-14^{\circ}$ and we have just shown that the latter case does not hold.

\subsection{Orientation of surface patches}

We will need the following technical lemma.

LEMma 17. Let $C_{1}$ and $C_{2}$ be two infinite cones with cone angle $\theta$, with the same apex $p$ and same axis, extended in opposite directions. Let $x$ be a point not in the interior of either of the convex hulls of $C_{1}$ or $C_{2}$. Consider a line $\ell$ passing through $x$, making an angle of $\alpha<\theta$ with the common axis of $C_{1}$ and $C_{2}$, and hitting $C$ and $C^{\prime}$ in points $x_{1}$ and $x_{2}$. Then we have

$$
\left\|x_{1}-x_{2}\right\| \leq 2 \cdot\|x-p\| \cdot \frac{\cos \theta}{\sin (\theta-\alpha)}
$$

Proof. Without loss of generality assume that $p$ is the origin and that the common axis of $C_{1}$ and $C_{2}$ is the $z$ axis. By the assumptions of the lemma, $x_{1}$ and $x_{2}$ are in opposite sides of $c$ on $\ell$. Consider the vertical hyperplane $H$ containing $x$ and the $z$ axis. It is easy to observe that $\left\|x-x_{1}\right\|$ would be maximized if $\ell$ was in $H$, in which case by the law of sines, we would get $\left\|x-x_{1}\right\| \leq\left\|x-y_{1}\right\| \cdot \sin \theta / \sin (\theta-\alpha)$, where $y_{1}$ is the vertical projection of $x$ to $C_{1}$. Similarly we would get $\left\|x-x_{2}\right\| \leq\left\|x-y_{2}\right\| \cdot \sin \theta / \sin (\theta-\alpha)$, where $y_{2}$ is the vertical projection of $x$ to $C_{2}$. On the other hand $\left\|y_{1}-y_{2}\right\|$ is maximized when $x p$ is horizontal (perpendicular to the $z$ axis) in which case we get $\left\|y_{2}-y_{1}\right\|=2 \cdot\|x-p\| \cot \theta$. Combining these, we get the desired bound.

Let $c$ be a surface 2-saddle. By definition, $c$ is the intersection point of a Delaunay triangle $t_{c}$ and its dual Voronoi edge $e_{c}$. Let $p$ be the vertex of $t_{c}$ with the largest angle. Notice that $\|p-c\|$ is the circumradius of $t_{c}$ and by Corollary $5,\|p-c\| \leq 1.6 \varepsilon f(p)$. Therefore by Lemma 14, the normal of $t_{c}$ makes an angle of at most $\beta(1.6 \varepsilon)=8 \varepsilon$ with normal to surface at $p$. In other words, $t_{c}$ lies flat to surface and it is therefore meaningful to distinguish between the side of it that faces the interior of $\Sigma$ and the one that faces its exterior. We refer to these sides of $t_{c}$ as its inner and outer sides respectively. Since $t_{c}$ intersects $e_{c}$ in a point of its relative interior (by our non-degeneracy assumption), we can distinguish between the two endpoints of $e_{c}$ as its inner and outer vertices and refer to them as $v_{c}^{-}$and $v_{c}^{+}$respectively. We denote by $e_{c}^{+}$, the the segment $c v_{c}^{+}$excluding $c$ and we define $e_{c}^{-}$similarly. Notice that the flow direction on $e_{c} \backslash\{c\}$ is toward its endpoints at each side of $c$. Therefore, every point of $e_{c}$ between $c$ and $v_{c}^{+}$flows to the same maximum that $v_{c}^{+}$ flows into. A similar statement holds for the points between $c$ and $v_{c}^{-}$. We define $U_{c}^{+}=e_{c}^{+} \cup \phi\left(v_{c}^{+}\right)$and $U_{c}^{-}=e_{c}^{-} \cup \phi\left(v_{c}^{-}\right)$. In fact, $U_{c}^{+}$and $U_{c}^{-}$together make the unstable manifold of $c$ [12]. Thus, if $U_{c}^{+}$intersects $\Sigma^{+}$then the flow originated at any point of $e_{c}^{+}$, arbitrarily close to $c$ must end up in an exterior medial axis maximum $m$ and therefore $S(c)$ is incident to $S(m)$ through the outer side of $t_{c}$. Similar statements can be made by replacing $U_{c}^{+}$with $U_{c}^{-}$and $\Sigma^{+}$with $\Sigma^{-}$.

Lemma 18. For any $0<\rho<1$, there exists $\varepsilon_{0}$ small enough such that if $P$ is an $\varepsilon$-sampling of $\Sigma$ for $\varepsilon \leq \varepsilon_{0}$, then for any $x \in U_{c}^{+} \cap \tilde{\Sigma}, \angle\left(v(x), n_{p}^{+}\right) \leq 12 \varepsilon$, and for every point $x \in U_{c}^{-} \cap \tilde{\Sigma}, \angle\left(v(x), n_{p}^{-}\right) \leq 12 \varepsilon$, where $p$ is any point in $A(x)$. In particular, for $\rho=\delta / \varepsilon \geq 3 / 4, \varepsilon_{0}=0.01$ suffices.

Proof. We only prove the lemma for points in $U_{c}^{+} \cap \tilde{\Sigma}$. The proof for points in $U_{c}^{-} \cap \tilde{\Sigma}$ is analogous. For simplicity we enforce $\varepsilon_{0} \leq 0.01$ although the statement of the lemma may hold for larger values of $\varepsilon_{0}$. Let $P$ be a $\varepsilon$-sampling of $\Sigma$ with $\varepsilon \leq \varepsilon_{0} \leq 0.01$.

Since $x \in \tilde{\Sigma} \subset \Sigma_{12 \varepsilon^{2}}$, by Corollary $5,\|x-p\| \leq 1.6 \varepsilon f(p)$ for every $p \in A(x)$. Notice that it suffices to prove that $\angle\left(v(x), n_{p}^{+}\right) \leq 8 \varepsilon$ for only one point $p \in A(x)$. This is because for any other point $q \in A(x),\|p-q\| \leq\|p-x\|+$ $\|q-x\| \leq 2 \cdot 1.6 \varepsilon \max \{f(p), f(q)\}$ and therefore by Lemma $1, \angle\left(n_{p}^{+}, n_{q}^{+}\right) \leq \frac{3.2 \varepsilon}{1-3 \cdot 3.2 \varepsilon} \leq 4 \varepsilon$ for $\varepsilon \leq 0.01$.

As above, let $t_{c}$ and $e_{c}$ be the Delaunay triangle and its dual Voronoi edge for which $\{c\}=t_{c} \cap e_{c}$. Notice that $U_{c}^{+}$is a piece-wise linear curve. Let $u_{0}, u_{1}, \ldots, u_{k}$ be the vertices of this curve with $u_{0}=c, u_{1}=v_{c}^{+}$, and $u_{k}=m$, where $m$, the maximum at which $U_{c}^{+}$ends. Notice of course that $v_{0}$ itself does not belong to $U_{c}^{+}$as $v\left(u_{0}\right)=v(c)=0$. We prove the lemma inductively starting from the segment $u_{0} u_{1}$ and going up to $u_{k-1} u_{k}$. By the structure of flow complex [12], it is easy to see that every vertex in $\left\{v_{1}, \ldots, v_{k}\right\}$ is either a Voronoi vertex or is on a Voronoi edge. Furthermore, the interior of every segments $u_{i-1} u_{i}, i=1, \ldots, k$, falls entirely inside a Voronoi edge or facet.

For the base case of our induction we observe that the lemma holds for points $x \in v_{0} v_{1}$ (excluding $v_{0}$ ). To see this, notice that the direction of $v(x)$ for such points agrees with the vector $v_{c}^{+}-c$. Let $p$ be the vertex of $t_{c}$ with the largest angle. Using Lemma 14, and taking into account that $x$ is on the outer side of $t$, implies that the angle between $n^{+}(p)$ and $v_{c}^{+}-c$ is at most $\beta(1.6 \varepsilon) \leq 8 \varepsilon$.

Notice that with a similar argument as for the case of the points on $e_{c}^{+}$, for any point $x \in U_{c}^{+}$that flows on a Voronoi edge $e$, the Delaunay triangle $t$ dual to $e$ must lie flat to surface and thus we can distinguish between its side facing outward and the one facing inward. Informally, we will say that in such a case $x$ is above of $t$ if $x$ is on the side of $t$ facing outward and below $t$ otherwise.

For the rest of the proof we consider all cases for the flow to move from one Voronoi face to another.

1. Edge-vertex-edge. First we study the case in which the flow on a Voronoi edge $e$, reaches a Voronoi vertex $v$ and enters another Voronoi edge $e^{\prime}$. We assume that the statement of the lemma holds for points on $e$ and show that it remains true as flow moves on to $e^{\prime}$. To see this, let $t=p q r$ 


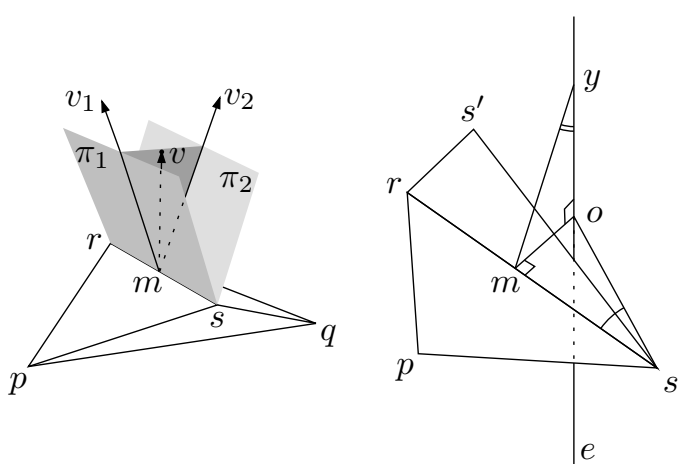

Figure 6.

be the Delaunay triangle dual to $e$ and let $t^{\prime}=q r s$ be the one dual to $e^{\prime}$. The Voronoi vertex $v$ must be dual to the Delaunay tetrahedron $\Delta$ with vertex set $\{p, q, r, s\}$. Since the flow through $v$ continues on $e^{\prime}$, the driver of flow for points $x \in e^{\prime}$ must lie in the interior of the triangle $t^{\prime}$ or in other words, the line through $e^{\prime}$ must intersect $t^{\prime}$. As discussed above both $t$ and $t^{\prime}$ are flat and $v$ is above $t$. It is easy to see that extending the lemma $e^{\prime}$ is identical to showing that $v$ is also above $e^{\prime}$. The outward direction for both $t$ and $t^{\prime}$ are within $12 \varepsilon$ from $n_{q}^{+}$. It can be easily observed that if $v$ is not above $t^{\prime}$, it must be in $\Delta$. But in that case $v$ is a maximum of the distance function and the flow does not leave it to enter $e^{\prime}$.

2. Facet-vertex-edge. The second case we study is that of the flow through a Voronoi facet $f$ dual to Delaunay edge $p q$ reaches a Voronoi vertex $v$ dual to tetrahedron $\Delta=p q r s$ and continues on a Voronoi edge $e$ dual to Delaunay triangle $t=q r s$. We assume that the lemma holds for the points $x$ on $U_{c}^{+} \cap f$ and show that this extends to the points on $e$. As in the previous case $t$ is flat and we only need to show that $v$ is above $t$. By induction hypothesis, $\angle\left(v-d, n_{q}^{+}\right) \leq 12 \varepsilon$ where $d=\frac{1}{2}(p+q)$ is the driver of the points on $f$. It can be verified that if $v$ is not above $t$, it must be that $v \in \Delta$ making $v$ a local maximum, a contradiction.

3. Edge-vertex-facet. Consider now the case where the flow through a Voronoi edge $e$ reaches a Voronoi vertex $v$ and enters a Voronoi facet $f$ incident to $v$. Let $p q r$ be Delaunay triangle dual to $e$ and let $r s$ be the Delaunay edge dual to $f$. Note that $v$ is the circumcenter of the Delaunay tetrahedron $\Delta=$ pqrs. We assume that the lemma holds for points on $e$ which is identical to assuming that $v$ is above $t$. Since the flow through $v$ continues on $f$, the closest point of $\Delta$ to $v$ is the midpoint $m=\frac{1}{2}(r+s)$ of the edge $r s$ (see Figure 6, left). For this to happen, $v$ must be in the wedge made by two half-planes $\pi_{1}$ and $\pi_{2}$ both having the line through $r s$ as boundary and respectively orthogonal to triangles $t_{1}=p r s$ and $t_{2}=q r s$. Since $v$ is the circumcenter of $\Delta,\|v-r\| \leq$ $1.6 \varepsilon f(r)$ is an upper bound for the circumradii of both $t_{1}$ and $t_{2}$. Similar to the argument in the previous case normals to both $t_{1}$ and $t_{2}$ make an angle of at most $12 \varepsilon$ with $n_{r}$. Since $v$ is above $t$ but not contained in $\Delta$, it must be above both $t_{1}$ and $t_{2}$. If we base at $m$, two vectors $v_{1}$ and $v_{2}$, respectively normal to $t_{1}$ and $t_{2}$ in their outward directions, $v_{1}$ will lye in $\pi_{1}$ and $v_{2}$ in $\pi_{2}$. The segment $v m$ is on the plane bisecting $r s$ and so are $v_{1}$ and $v_{2}$. It follows from triangle inequality on angles between vectors that $v-m=v(x)$, for $x \in f \cap U_{c}^{+}$, also makes and angle of at most $12 \varepsilon$ with $n_{r}^{+}$. Notice that with exactly the same argument but with using $s$ instead of $r$, we get the same bound with respect to $n_{s}^{+}$.

4. Facet-vertex-facet. The proof of this case is a simple combination of the proofs of cases 2 and 3 .

5. Facet-edge-facet. We show now that under tight enough sampling, i.e. by choosing $\rho$ large enough, if the flow through a Voronoi facet $f$ arrives at a Voronoi edge $e$ of $f$, it will continue on $e$ and does not enter an other facet $f^{\prime}$ incident to $e$, given that the statement of lemma holds for the points of $U_{c}^{+} \cap f$ (we give a proof for $\rho \geq 3 / 4$ but better bounds can be achieved for smaller $\varepsilon$ or through slicker analysis). Suppose to the contrary that this is not the case, i.e. (see Figure 6, right) the flow crosses $e$ and enters another facet $f^{\prime}$ incident to $e$. Let $r s$ be the Delaunay edge dual to $f$. The driver of the flow on $f$ is $m=\frac{1}{2}(r+s)$. Let $y$ be the point where the flow reaches $e$. The dual Delaunay triangle $t$ to $e$ has $r$ and $s$ for vertices plus another vertex $s^{\prime}$. For the flow to cross $e$ and enter $f^{\prime}, f^{\prime}$ must be the Voronoi facet dual to $s s^{\prime}$. Furthermore, the line containing $e$ must not intersect $t$. Let $o$ be the circumcenter of $t$. By our assumption, the flow direction on $f$, which coincides with $m-y$ makes an angle of no more than $12 \varepsilon$ with $n_{r}^{+}$. On the other hand, $y \in \tilde{\Sigma}$ and thus $t$ is flat and since $r$ is the largest angle in $t$, the normal to $t$, i.e. direction of $e$, makes an acute angle of $8 \varepsilon$ with $n_{r}$. This in particular implies that $y$ is above $t$. Therefore, $\angle m y o$ is at most $20 \varepsilon$. In order for the line containing $e$ not to intersect $t$, it must hold that $\angle m s s^{\prime}<\angle m s o$. The two triangles mso and myo both share the edge $m o$ and both have a right angle on one of the end-points of this edge. We will show below that $\|m-w\|<\|m-s\|<\|o-y\|$. This will imply that $\angle m s o<\angle m y o$. We have already seen that $\angle m y o \leq 20 \varepsilon$ and using exactly the same argument as in the proof of Lemma 15 , we get $\angle m s o \geq \arcsin (\rho / 3.2)$, and therefore we must have

$$
20 \varepsilon>\arcsin \left(\frac{\rho}{3.2}\right) .
$$

This inequality is violated for $\varepsilon \leq \varepsilon_{0}$ for small enough $\varepsilon_{0}$ (in particular for $\varepsilon_{0}=0.01$ and $\rho \geq 3 / 4$ ) giving us the desired contradiction. Now to prove that $\|m-y\| \leq\|m-s\|$. Notice that $s$ is a closest sample to $y$ and by our assumption $y \in \tilde{\Sigma}$. Therefore, $y$ is between the cones $C_{s}^{+}$and $C_{s}^{-}$. On the other hand by Corollary $5,\|r-s\|=2\|m-s\| \leq 2\|y-s\| \leq$ $2 \cdot 1.6 \varepsilon f(s)$ and therefore using Lemma 13, ms makes an angle of at least $\frac{\pi}{2}-1.6 \varepsilon>\theta$ with normal to $\Sigma$ at $s$. This implies that $m$ is also between $C_{s}^{+}$and $C_{s}^{-}$. By our inductive hypothesis, my makes an angle of at most $12 \varepsilon$ with $n_{s}^{+}$. Lemma 17 can now be used to get

$$
\|m-y\| \leq 2 \cdot 1.6 \varepsilon \cdot f(s) \frac{\sin (5 \varepsilon)}{\cos (17 \varepsilon)}<\frac{1}{2} \delta f(s) \leq\|m-s\|,
$$

where the middle inequality holds for $\varepsilon \leq 0.01$ and $\rho \geq 3 / 4$. In fact for any constant $0<\rho<1$ the above inequality holds (and the desired contradiction is achieved) for any $\varepsilon \leq \varepsilon_{0}$ for small enough $\varepsilon_{0}$ since the left hand side has a quadratic dependence on $\varepsilon$.

Thus we have proved that whenever the flow on $U_{c}^{+}$moves to a Voronoi facet $f$, it leaves $f$ by either hitting a Voronoi edge $e$ and continuing on $e$, or by hitting a vertex $v$. Thus we have covered all cases in the inductive step and this completes the 
proof of the lemma.

In the following Lemma we show that if $S(c)$ is incident to the stable manifold $S(m)$ of an interior (exterior) medial axis maximum $m$, then the part of $S(c)$ that is contained in $t_{c}$ is incident to $S(m)$ at the inner (outer) side of $t_{c}$.

Lemma 19. For any surface 2-saddle $c, U_{c}^{+}$can only intersect with $\Sigma^{+}$and $U_{c}^{-}$can only intersect with $\Sigma^{-}$.

Proof. We prove the claim for $U_{c}^{+}$. The other claim is proved analogously. Suppose to the contrary that $U_{c}^{+}$intersects $\Sigma^{-}$at $x$. Let $v$ be the last turning point of $U_{c}^{+}$before reaching $x$. Let $q$ be a sample point for which $x \in C_{q}^{-}$and let $p$ be a closest sample point to $x$. Then $\|x-p\| \leq\|x-q\| \leq$ $f(q) \cos \theta=f(q) \sin (5 \varepsilon)$. Therefore, $\|p-q\| \leq 2 f(q) \sin (5 \varepsilon)$ and as such by Lemma 1 ,

$$
\angle\left(n_{p}^{+}, n_{q}^{+}\right) \leq \frac{2 \sin (5 \varepsilon)}{1-3 \cdot 2 \sin (5 \varepsilon)}=O(\varepsilon) .
$$

On the other hand, by Lemma 18, the vector $x-v$ makes an angle of $O(\varepsilon)$ with $n_{p}^{+}$. It is easy to observe that this contradicts the assumption that the flow hits $C_{q}^{-}$.

The following lemma is a direct consequence of Lemma 18 and Lemma 16.

LEMMA 20. Let $c_{1}$ and $c_{2}$ be two surface critical points with $S\left(c_{1}\right)$ and $S\left(c_{2}\right)$ put by RECONSTRUCT into $\mathcal{T}$, such that boundaries of $S\left(c_{1}\right)$ and $S\left(c_{2}\right)$ have a Gabriel edge $e$ in common. Let $t_{1}$ and $t_{2}$ be the patch triangles incident to $e$ in $S\left(c_{1}\right)$ and $S\left(c_{2}\right)$, respectively. Then, the dihedral angle between $t_{1}$ and $t_{2}$ is greater than $\pi / 2$.

Proof. Orient patch triangles of $S\left(c_{1}\right)$ by taking for every patch triangle $t$ of $S\left(c_{1}\right)$, the normal to $t$ pointing to the side of $t$ incident to the interior of the reconstruction as $n_{t}^{-}$. Lemma 18 implies that in this case for every point $x \in t$ where $t$ is a patch triangle of $S\left(c_{1}\right), \angle\left(n_{\hat{x}}^{+}, n_{t}^{+}\right)<14^{\circ}$. In particular, by letting $t=t_{1}$ and choosing $x$ to be a point on $e$, we get $\angle\left(n_{\hat{x}}^{+}, n_{t_{1}}^{+}\right)<14^{\circ}$

If we do a similar orientation on $S\left(c_{2}\right)$, we get $\angle\left(n_{\hat{x}}^{+}, n_{t_{2}}^{+}\right)<$ $14^{\circ}$. As such, the dihedral angle between $t_{1}$ and $t_{2}$ is at least $180^{\circ}-28^{\circ}=152^{\circ}$.

\subsection{Homeomorphism}

THEOREM 3. The output $\mathcal{T}$ produced by the algorithm RECONSTRUCT is a 2-manifold without boundary homeomorphic to $\Sigma$.

Proof. First we observe that the complex $\mathcal{T}$ produced by RECONSTRUCT is the boundary of the union of stable manifolds of either the inner or outer medial axis critical points. Let $m_{1}$ and $m_{2}$ be medial axis maxima such that $S\left(m_{1}\right)$ and $S\left(m_{2}\right)$ are neighboring 3-cells in the flow complex, i.e. they both have $S(c)$ contained in their boundaries, where $c$ is a 2 -saddle. If $m_{1}$ is an inner medial axis maximum and $m_{2}$ an outer one, then $c$ must be a surface critical point as the common boundary of $S\left(m_{1}\right)$ and $S\left(m_{2}\right)$ must lie in $\tilde{\Sigma} \subset \Sigma_{12 \varepsilon^{2}}$. On the other hand, if $m_{1}$ and $m_{2}$ are both inner (outer) medial axis maxima then $S(c)$ cannot be a surface critical point since otherwise both $U_{c}^{+}$and $U_{c}^{-}$arrive at inner (outer) medial axis maxima and therefore both must have crossed $\Sigma^{-}$ $\left(\Sigma^{+}\right)$and this violates Lemma 19. This in part implies that the algorithm RECONSTRUCT in fact partitions the medial axis critical points into two subsets.

We consider in this proof the case where $\mathcal{T}$ is the boundary of the union of stable manifolds of the inner medial axis critical points (the outer case being analogous). We argue that $\mathcal{T}$ and $\Sigma$ are homeomorphic. Consider the restriction $\zeta: \mathcal{T} \rightarrow \Sigma$ of the closest point map $x \mapsto \hat{x}$. We prove that $\zeta$ is a homeomorphism. Since both $\mathcal{T}$ and $\Sigma$ are compact, it is sufficient to show that $\zeta$ is continuous, one-to-one and onto.

First, we argue that $\zeta$ is one-to-one. Orient the normal to each patch triangle $t$ so that it makes an angle less than $\frac{\pi}{2}$ with the oriented normal $n_{p}^{+}$at the vertex $p$ of $t$ which is a sample point. Because of Lemma 16 and Lemma 20, the triangles of $\mathcal{T}$ can be oriented consistently satisfying this condition. We denote this oriented normal for a patch triangle $t$ by $n_{t}$. By Corollary 6 , for every point $x$ in a patch triangle $t$ the oriented triangle normal $n_{t}$ makes an angle of at most $14^{\circ}$ with $n_{\hat{x}}^{+}$. Suppose $\zeta$ is not one-to-one. Then, there are two points $x$ and $x^{\prime}$ in $\mathcal{T}$ that are both mapped to the same point $\hat{x}$ by $\zeta$. Consider the line $\ell$ normal to $\Sigma$ at $\hat{x}$. This line passes through both $x$ and $x^{\prime}$. Assume without loss of generality that $x$ and $x^{\prime}$ are consecutive intersection points of $\ell$ and $\mathcal{T}$. Then, at one of $x$ and $x^{\prime}$ the line $\ell$ enters and at the other exits the interior bounded by $\mathcal{T}$. In other words, if we orient $\ell$ along $n_{\hat{x}}^{+}$, it makes an angle at least $\frac{\pi}{2}$ with one of the oriented normals of $\mathcal{T}$ at $x$ or $x^{\prime}$, an impossibility.

Next, we argue that $\mathcal{T}$ is a manifold. Since $\mathcal{T}$ bounds the union of the closed stable manifolds of medial axis maxima, it is a 2-complex with each edge being incident to at least two triangles. We claim that the triangles incident to each vertex $v$ of this complex form a topological disk and hence $\mathcal{T}$ is a 2-manifold. If not, there are two triangles incident to $v$ so that a normal line stabs both of them at points arbitrarily close to $v$ since they lie almost parallel to $\Sigma$. This is in contradiction with $\zeta$ being one-to-one.

We are left to show that $\zeta$ is continuous and onto. The continuity of $\zeta$ follows from the fact that the original closest point function $x \mapsto \hat{x}$ is continuous everywhere except at the medial axis. To show that $\zeta$ is onto, consider $\zeta(\mathcal{T}) \subseteq \Sigma$. Certainly, $\zeta$ is onto from $\mathcal{T}$ to $\zeta(\mathcal{T})$. We claim that $\zeta(\mathcal{T})=\Sigma$. Since $\mathcal{T}$ is a 2 -manifold without boundary and $\zeta$ maps it homeomorphically to $\zeta(\mathcal{T})$, we have $\zeta(\mathcal{T})$ as a compact 2manifold without boundary and $\zeta(\mathcal{T}) \subseteq \Sigma$. This is only possible if $\zeta(\mathcal{T})=\Sigma$ as both $\zeta(\mathcal{T})$ and $\Sigma$ are compact 2manifolds without boundary.

\section{CURVES IN $\mathbb{R}^{3}$}

\subsection{Separation of critical points}

Let $P$ be an $\varepsilon$-sample of a smooth closed curve $\Gamma \subset \mathbb{R}^{3}$. We analyze the critical points of the distance function $h$ induced by $P$.

Lemma 4 still holds, i.e., all critical points of $h$ are either near the curve (called the curve critical points), or near the medial axis (called the medial axis critical points). However, 
unlike surfaces, not all types of critical points can be near the curve.

Lemma 21. If the boundary of a ball $B$ intersects $\Gamma$ in three or more points, then it contains a medial axis point.

Proof. Shrink $B$ centrally untill its boundary becomes tangent to a point, say $x$ of $\Gamma$. Then keeping $x$ fixed on the boundary shrink it further by moving its center toward $x$. Stop when the interior of $B$ becomes empty of $\Gamma$. At this moment $B$ is tangent to $\Gamma$. If $x$ is the only point of tangency, $B$ is a curvature ball and its center is on the medial axis. If it is tangent to two or more points of $\Gamma$, its center is again a medial axis point. In both cases the medial axis point is in the original ball $B$.

LEMmA 22. Let $c$ be a critical point of $h$. If $c \in \Gamma_{\varepsilon^{2}}$, then $c$ is either an index-0 or an index- 1 critical point provided that $\varepsilon<1 / 3$.

Proof. If $c$ is an index- 2 or index- 3 critical point, we have a ball $B$ centered at $c$ whose boundary intersects $\Gamma$ in three or more points. Let $r$ be the radius of $B$. By Lemma 21, $B$ contains a medial axis point and hence $r>f(p)$ for any point $p \in P \cap B$. Therefore, $c$ is at least $f(p)$ distance away from its closest point in $P$. We claim that $c$ is also at least $\varepsilon^{2} f(\hat{c})$ distance away from $\hat{c}$ proving that $c \notin \Gamma_{\varepsilon^{2}}$.

To reach a contradiction assume that $s(c)=\|c-\hat{c}\|$ is no more than $\varepsilon^{2} f(\hat{c})$. The closest sample point, say $p$, to $\hat{c}$ is within $\varepsilon f(\hat{c})$ distance from it. This point $p$ is within $(\varepsilon+$ $\left.\varepsilon^{2}\right) f(\hat{c})$ distance from $c$. Applying the Lipschitz property of the feature size $f$ we get that $\|p-c\| \leq \frac{\varepsilon+\varepsilon^{2}}{1-\varepsilon-\varepsilon^{2}} f(p)$. On the other hand we know $\|p-c\|>f(p)$. Thus we reach a contradiction if $\varepsilon<\frac{1}{3}$.

\subsection{Reconstruction}

We will state some more results regarding the critical points of the distance from a curve. These results lead straightforwardly to a reconstruction algorithm. The edges that connect two consecutive points on $\Gamma$ are called correct edges. All other edges are incorrect. It is known that all correct edges are Delaunay if $\varepsilon<1 / 3$. Also, it is easy to show that they intersect their dual Voronoi facets, i.e., they contain index-1 critical points. It is further known that the length of any correct edge $p q$ is at most $\frac{2 \varepsilon}{1-\varepsilon} f(p)$. This means the index-1 critical point which is the midpoint of $p q$ is at most $\frac{\varepsilon}{1-\varepsilon} f(p)$ distance away from its closest sample point which suggests that this critical point cannot lie in $M_{2 \varepsilon}$ and hence resides in $\Gamma_{\varepsilon^{2}}$. On the other hand, as the next lemma shows, the incorrect edges containing index-1 critical points are longer.

Lemma 23. Let $p q$ be a Delaunay edge that contains an index-1 critical point $c$.

(i) If $p q$ is correct then the distance of $c$ from $p$ is at at most $\frac{\varepsilon}{1-\varepsilon} f(p)$.

(ii) If $p q$ is incorrect then the distance of $c$ from $p$ is at least $f(p) / 2$.

Proof. If $p q$ is correct, its length is at most $\frac{2 \varepsilon}{1-\varepsilon} f(p)$ by Lemma 3.4 of [9]. So, its midpoint $c$ has distance at most $\frac{\varepsilon}{1-\varepsilon} f(p)$ from $p$ proving (i).
Now assume that $p q$ is incorrect. If the boundary of the ball $B$ centered at $c$ with radius $\|c-p\|$ intersects $\Gamma$ only in $p$ and $q$ then $B$ is a medial ball and the distance of $c$ from $p$ is at least $f(p)$. Otherwise the boundary of $B$ intersects $\Gamma$ in more than two points and hence $B$ contains a medial axis point by Lemma 21 . Therefore, the diameter of $B$ is at least $f(p)$ and the distance of $c$ from $p$ is at least $f(p) / 2$ proving (ii).

The curve critical points can be separated from the medial critical points using an algorithm similar to the one for surfaces. For a sample point $p$ we determine the nearest critical point. By Lemma 22 and Lemma 23 this critical point has index 1 and is the midpoint of a correct edge. A result of Amenta et al. [2] implies that a correct edge makes an angle of at most $\arcsin (\varepsilon / 2)$ with the tangent $t_{p}$ at $p$. Thus the vector from $p$ to its nearest critical point makes an angle no larger than $\arcsin (\varepsilon / 2)$ with $t_{p}$. On the other hand any index-1 critical point in $M_{2 \varepsilon}$ is at least $f(p) / 2$ distance away from $p$ as a result of Lemma 23. This fact along with the following result due to Dey et al. [8] give the required critical point separation. For a point $p \in \Gamma$, the space spanned by the vectors normal to $t_{p}$ is called its normal space.

Lemma 24. Let $x$ be a point in $V_{p}$ where $\|p-x\| \geq \delta f(p)$. Then there is a vector $v_{p}$ in the normal space of $p$ so that $\angle\left(v_{p}, x-p\right) \leq \arcsin \frac{\varepsilon}{\delta(1-\varepsilon)}+\arcsin \frac{\varepsilon}{1-\varepsilon}$.

Combining the results of [2], Lemma 23 and Lemma 24 we get the following corollary.

Corollary 7. Let $c$ be any index-1 critical point on an edge pq. Let $c^{\prime}$ be the nearest index-1 critical point of $p$. Then, for $\varepsilon<1 / 3$

$$
\begin{aligned}
& \text { (i) } \angle\left(c^{\prime}-p, c-p\right)>\pi / 4 \text { if } p q \text { is incorrect, and } \\
& \text { (ii) } \angle\left(c^{\prime}-p, c-p\right)<\pi / 4 \text { if } p q \text { is correct. }
\end{aligned}
$$

We get an immediate separation algorithm for index-1 critical points which also gives a curve reconstruction algorithm. For each point $p$ determine the shortest Gabriel edge. Let this edge be $p q$. Choose the other Gabriel edge incident to $p$ which makes less than $\pi / 4$ angle with $p q$. These are the two correct edges for $p$.

\section{CONCLUSION}

We provide the first theoretical results that link the critical points of the distance function to an $\varepsilon$-sampling of a curve or surface embedded in $\mathbb{R}^{3}$ to either the surface (curve) or its medial axis. This allows us to derive reconstruction algorithms for curves and surfaces embedded in $\mathbb{R}^{3}$ that come with topological and geometric reconstruction guarantees. Although our proofs of normal convergence and because of that topological correctness depend on the assumption that the given $\varepsilon$-sampling is tight, we believe that these guarantees can be strengthened to the case of general $\varepsilon$-sampling.

The output of our algorithm is not a Delaunay subcomplex, a property sometimes desired in practice. However, the output of the algorithm can be easily modified to satisfy this requirement. A natural way is to replace the stable 
manifold of a surface 2-saddle with the union of Delaunay triangles corresponding to its flow complex triangles (See Appendix A). Notice that doing this, we replace each patch with a patch made of the Delaunay triangles which shares the same boundary of Gabriel edges with the original patch. Furthermore, these triangles are asymptotically as close to the surface as our reconstruction. Another provable method that uses the ideas applied in this paper and is related to the heuristic method of construction of flow complex used in [7] is given in Appendix B.

\section{References}

[1] N. Amenta and M. Bern. Surface reconstruction by Voronoi filtering. Discr. Comput. Geom., 22, pp. 481504, (1999).

[2] N. Amenta, M. Bern and D. Eppstein. The crust and the beta-skeleton: combinatorial curve reconstruction. Graphical Models and Image Processing, 60, pp. 125135 (1998).

[3] N. Amenta, S. Choi, T. K. Dey and N. Leekha. A simple algorithm for homeomorphic surface reconstruction. Internat. J. Comput. Geom. \& Applications, vol. 12, 2002, pages $125-141$.

[4] N. Amenta, S. Choi and R. Kolluri. The power crust, unions of balls, and the medial axis transform. Computational Geometry: Theory and Applications, 19, pp. 127-153, (2001).

[5] J. D. Boissonnat and F. Cazals. Smooth Surface Reconstruction via Natural Neighbour Interpolation of Distance Functions. Computational Geometry: Theory and Applications, 22, pp. 185-203, (2002).

[6] R. Chaine. A geometric convection approach of 3-D reconstruction. In Proc. Eurographics Sympos. on Geometry Processing, pp. 218 - 229, (2003).

[7] T. K. Dey, J. Giesen and S. Goswami. Shape Segmentation and Matching with Flow Discretization. In Proc. 8th Workshop on Algorithms Data Strucutres, pp. 25$36,(2003)$.

[8] T. K. Dey, J. Giesen, S. Goswami and W. Zhao. Shape dimension and approximation from samples. Discr. Comput. Geom., 29, pp. 419-434 (2003).

[9] T. K. Dey and P. Kumar. A simple provable algorithm for curve reconstruction. In Proc. 10th Annual ACMSIAM Symposium on Discrete Algorithms, pp. 893-894, (1999).

[10] T. K. Dey and W. Zhao. Approximating the Medial Axis from the Voronoi Diagram with a Convergence Guarantee. Algorithmica, 38, pp. 179-200 (2004)

[11] H. Edelsbrunner. Surface reconstruction by wrapping finite point sets in space. Discr. Comput. Geom., 32, pp. 231-244, (2004)

[12] J. Giesen and M. John. The Flow Complex: A Data Structure for Geometric Modeling. In Proc. 14th Annual ACM-SIAM Symposium on Discrete Algorithms, pp. 285-294, (2003)

[13] K. Grove. Critical Point Theory for Distance Functions. In Proceedings of Symposia in Pure Mathematics 54(3), pp. 357-385, (1993)

\section{APPENDIX}

\section{A. Stable manifolds of index-2 critical points}

An index-2 critical point, i.e., a saddle point $s$, is the intersection point of a Delaunay triangle $t$ with its dual Voronoi edge $e$. Under a mild non-degeneracy condition, the stable manifold of $s$ is a surface patch that can be constructed explicitly, see [12]. The degeneracy condition is that the inflow of $s$ does not contain a Voronoi vertex which can be always achieved by an arbitrarily small perturbation of the sample points. We start by constructing a polygon $P$ whose interior points all flow into $s$. This polygon contains $s$ and is contained in $t$. To simplify our exposition assume that there are three Voronoi facets incident to every Voronoi edge. We are going to construct a polyline for each of the three Voronoi facets incident to $e$. These three polylines together make up the boundary of the polygon $P$. The drivers of the Voronoi facets incident to $e$ are points on their dual Delaunay edges. These Delaunay edges are all in the boundary of $t$. Note that it is possible that such a driver is a index- 1 critical point. First, consider a driver $d$ which is not an index-1 critical point. The line segment that connects $d$ with $s$ is contained in $t$ and intersects the boundary of the corresponding Voronoi facet in two points, namely in $s$ and in a second point $s^{\prime}$. We get a polyline from the two segments that connect $s^{\prime}$ to the two Delaunay vertices incident to the Delaunay edge that contains $d$. Second, if the driver of the Voronoi facet is a saddle of index-1 we take its dual Delaunay edge as the polyline. That is, we get three polylines all contained in $t$, one for each Voronoi facet incident to $e$. Let $P$ be the polygon whose boundary is given by these polylines. $P$ is contained in $t$ and all its interior points flow into $s$. It can be triangulated by connecting $s$ with the points $s^{\prime}$ and the Delaunay vertices incident to $t$. Figure 7 shows two examples of two such polygons $P$.
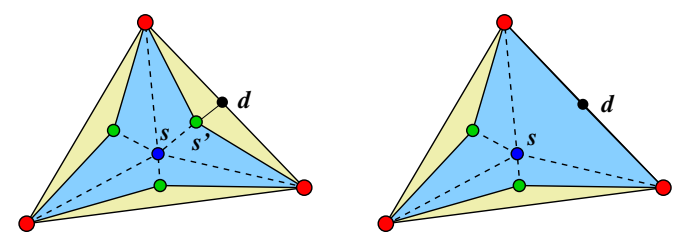

Figure 7. Two examples of polygons that are contained in a Delaunay triangle that is intersected by its dual Voronoi edge in $s$. The interior points of these polygons flow into $s$. The polygon in the figure on the right has one index- 1 critical point on its boundary.

Let $s^{\prime}$ be a point as constructed above for a Voronoi facet that is not driven by an index- 1 critical point. By construction $s^{\prime}$ is contained in a Voronoi edge $e^{\prime}$. Furthermore, by our assumption it has to be an interior point of $e^{\prime}$. We can assume again that $e^{\prime}$ is incident to three Voronoi facets. For one of these Voronoi facets we have already computed a polyline. For the remaining two we do it exactly the same way we did it above for $P$. Thus we have again three polylines, one for each Voronoi facet incident to $e^{\prime}$. Two of these polylines always intersect in a common Delaunay vertex. That is, the three polylines together form a polyline which is homeomorphic to $\mathbb{S}^{1}$. The latter polyline need not be contained in a hyperplane but it can be triangulated by connecting the 
point $s^{\prime}$ with newly computed points $s^{\prime}$ and to the Delaunay vertices incident to the Delaunay facet dual to $e^{\prime}$. This gives us a new triangulated surface patch whose interior points all flow into $s$.

We continue with the above construction until there are no more points $s^{\prime}$ left for which we have not already constructed a surface patch. The surface of points that flow into the index-2 saddle $s$ is made up from all the patches. By construction the boundary of this surface consists of Gabriel edges, i.e. Delaunay edges. Figure 8 shows an example of the stable manifold of some index- 2 critical point.

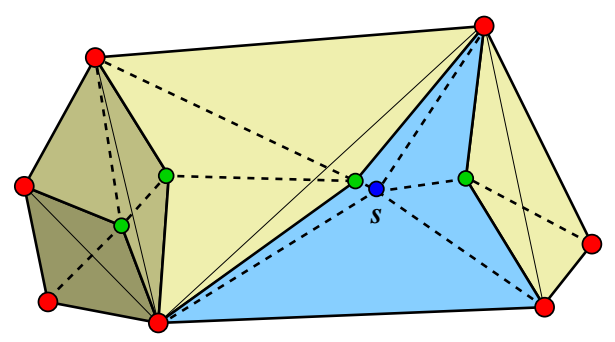

Figure 8. In this example the stable manifold of $s$ is made up from five surface patches. Note that the surface patches need not be planar.

\section{B. Delaunay subcomplex}

The flow complex is not a subcomplex of the Delaunay triangulation. Often a Delaunay mesh is preferred over other ones. So, we describe here briefly an algorithm to compute a Delaunay 2-complex whose Hausdorff distance to $\Sigma$ is small. The following definition becomes handy to describe and analyze the algorithm.

Definition 1. A Delaunay triangle $t$ is almost critical if the plane of $t$ intersects the dual Voronoi edge of $t$. The intersection point is called an almost critical point.

This algorithm approximates the stable manifolds of the medial axis maxima with an union of Delaunay tetrahedra. It processes these maxima in the order of their distances to $P$. Let $c$ be a medial axis maximum (critical point of index $3)$ to be processed. Let $\tilde{S}(c)$ denote the approximation of $S(c)$ which we compute. The algorithm initializes $\tilde{S}(c)$ with the Delaunay tetrahedron containing $c$ and collects adjacent Delaunay tetrahedra iteratively as follows: Let $t$ be any not almost critical triangle on the boundary of the union of tetrahedra collected so far. Let $\sigma$ and $\sigma^{\prime}$ be the two tetrahedra incident to $t$ out of which $\sigma$ is already in the collection for $c$. If $\sigma^{\prime}$ is not already in the approximate stable manifold of another maximum $x^{\prime}$, then $\sigma$ is put into $\tilde{S}(c)$. The following lemma guarantees that, at the termination $\tilde{S}(c)$ has only almost critical triangles on the boundary.

Lemma 25. If $t \in \partial \tilde{S}(c)$, then $t$ is almost critical.

Proof. The triangle $t$ can be on $\partial \tilde{S}(c)$ for the following reasons: (i) $t$ is almost critical, (ii) one tetrahedron incident to $t$ already belongs to $\tilde{S}\left(c^{\prime}\right)$ for a maximum $c^{\prime} \neq c$. There is nothing to prove in (i). So, consider (ii). The tetrahedron incident to $t$ and collected for $\tilde{S}(c)$ could not be in $\tilde{S}\left(c^{\prime}\right)$ only because $t$ is almost critical.

The output Delaunay 2-complex $T$ is the boundary of the union of $\tilde{S}(c)$ for all medial axis maxima c. By Lemma 25, all triangles in $T$ are almost critical. We show that they all lie close to $\Sigma$ and their unoriented normals agree with the surface normals.

First, we observe that the almost critical points have similar properties as the critical ones in that they also lie either near the surface $\Sigma$ or near the medial axis $M$. The proof follows the proof of Corollary 2.

Lemma 26. Let a be an almost critical point. For $\varepsilon<1 / 3$, either $a \in \Sigma_{\varepsilon^{2}}$ or $a \in M_{2 \varepsilon}$.

Now we argue that $T$ does not have any triangle $t$ whose almost critical point resides in $M_{2 \varepsilon}$.

Lemma 27. Let $t$ be a triangle in $T$. The circumcenter of $t$ is not in $M_{2 \varepsilon}$ for $\varepsilon<\min \{1 / 3, \sqrt{2 \Delta}\}$ where $\Delta=$ $\inf _{x, x^{\prime} \in \Sigma} \frac{f(x)}{f\left(x^{\prime}\right)}$.

Proof. Suppose on the contrary, the point $a$ where the plane of $t$ and its dual Voronoi edge intersect resides in $M_{2 \varepsilon}$. Let $t$ belong to $\tilde{S}(c)$. The tetrahedron incident to $t$ which is not in $\tilde{S}(c)$ is included in some approximate stable manifold, say $\tilde{S}\left(c^{\prime}\right)$. We claim that $c^{\prime}$ is a medial maximum and thus $t$ cannot be in the boundary of the union $\bigcup \tilde{S}_{x}$ for all medial maxima $x$.

The distance of $a$ from $P$ is at least $(1-2 \varepsilon) \mu(a)$. Since the maximum $c$ is further away from $P$ than $a$ is, we have $c^{\prime}$ at least $(1-2 \varepsilon) \mu(a)$ distance away from $P$. If $(1-2 \varepsilon) \mu(a)>$ $\varepsilon^{2} f\left(\hat{c}^{\prime}\right)$ the point $c^{\prime}$ has to be a medial axis maximum. The condition is satisfied for $\varepsilon<\sqrt{2 \Delta}$.

Since the circumcenter of each triangle in $T$ is in $\Sigma_{\varepsilon^{2}}$, their circumradius is small. The normals of such triangles are almost parallel to the surface normals due to Theorem 5 of Amenta et al. [3].

Lemma 28. Let $n_{t}$ be the normal of any triangle $t \in T$. Then the acute angle between the line of $n_{t}$ and the surface normal at any vertex of $t$ is $O(\varepsilon)$ when $\varepsilon$ is sufficiently small. 